\title{
Shapes of primordial non-Gaussianities in the Horndeski's most general scalar-tensor theories
}

\author{
Antonio De Felice ${ }^{1,2}$ and Shinji Tsujikawa ${ }^{3}$ \\ ${ }^{1}$ ThEP's CRL, NEP, The Institute for Fundamental Study, \\ Naresuan University, Phitsanulok 65000, Thailand \\ ${ }^{2}$ Thailand Center of Excellence in Physics, Ministry of Education, Bangkok 10400, Thailand \\ ${ }^{3}$ Department of Physics, Faculty of Science, Tokyo University of Science, \\ 1-3, Kagurazaka, Shinjuku-ku, Tokyo 162-8601, Japan
}

(Dated: August 16, 2018)

\begin{abstract}
In the Horndeski's most general scalar-tensor theories, we derive the three-point correlation function of scalar non-Gaussianities generated during single-field inflation in the presence of slowvariation corrections to the leading-order term. Unlike previous works, the resulting bispectrum is valid for any shape of non-Gaussianities. In the squeezed limit, for example, this gives rise to the same consistency relation as that derived by Maldacena in standard single-field slow-roll inflation. We estimate the shape close to the squeezed one at which the effect of the term inversely proportional to the scalar propagation speed squared begins to contribute to the bispectrum. We also show that the leading-order bispectrum can be expressed by the linear combination of two convenient bases whose shapes are highly correlated with equilateral and orthogonal types respectively. We present concrete models in which the orthogonal and enfolded shapes can dominate over the equilateral one.
\end{abstract}

\section{INTRODUCTION}

The potential presence of primordial non-Gaussianities in the CMB temperature anisotropies can be a powerful probe for the physics in the early Universe-especially for inflation [1]. The inflationary paradigm generally predicts nearly scale-invariant density perturbations [2] with a suppressed tensor-to-scalar ratio, whose prediction is consistent with the CMB power spectrum measured by COBE [3] and WMAP [4, 5]. The detection of scalar non-Gaussianities not only breaks the degeneracy among many inflationary models, but it also offers the possibility to discriminate between the inflationary paradigm and other alternative scenarios (such as curvaton [6]) [7]-[34].

There are several different shapes of non-Gaussianities depending on the wave numbers $\boldsymbol{k}_{1}, \boldsymbol{k}_{2}$, and $\boldsymbol{k}_{3}$ satisfying the condition $\boldsymbol{k}_{1}+\boldsymbol{k}_{2}+\boldsymbol{k}_{3}=0$ [35]-[45]. The simplest one is the so-called local shape, which has a peak in the squeezed limit (i.e., the limit where the modulus of the momenta approaches $k_{3} \rightarrow 0$ and $k_{1} \simeq k_{2}$ ). The second shape corresponds to the equilateral configuration with a peak at $k_{1}=k_{2}=k_{3}$. A factorizable shape whose scalar product with the equilateral template vanishes is called the orthogonal one. There is another shape dubbed the enfolded one, which is a linear combination of the equilateral and orthogonal templates.

From the bispectrum $\mathcal{A}_{\mathcal{R}}$ of the three-point correlation function of curvature perturbations $\mathcal{R}$, the non-linear parameter characterizing the strength of non-Gaussianities is defined by $f_{\mathrm{NL}}=(10 / 3) \mathcal{A}_{\mathcal{R}} / \sum_{i=1}^{3} k_{i}^{3}$. For purely adiabatic Gaussian perturbations we have that $f_{\mathrm{NL}}=0$, but the presence of non-Gaussian perturbations leads to the deviation from $f_{\mathrm{NL}}=0$. The WMAP 9 year data provide the following bounds on the non-linear parameters of local, equilateral, and orthogonal non-Gaussianities, respectively [46]:

$$
\begin{array}{llll}
f_{\mathrm{NL}}^{\text {local }}=37.2 \pm 19.9 & (68 \% \mathrm{CL}), & f_{\mathrm{NL}}^{\text {local }}=37 \pm 40 & (95 \% \mathrm{CL}), \\
f_{\mathrm{NL}}^{\text {equil }}=51 \pm 136 & (68 \% \mathrm{CL}), & f_{\mathrm{NL}}^{\text {equil }}=51 \pm 272 & (95 \% \mathrm{CL}), \\
f_{\mathrm{NL}}^{\text {ortho }}=-245 \pm 100 & (68 \% \mathrm{CL}), & f_{\mathrm{NL}}^{\text {ortho }}=-245 \pm 200 & (95 \% \mathrm{CL}) .
\end{array}
$$

Since the non-linear parameter of the enfolded shape is given by $f_{\mathrm{NL}}^{\text {enfold }}=\left(f_{\mathrm{NL}}^{\text {equil }}-f_{\mathrm{NL}}^{\text {ortho }}\right) / 2$ [44, 45], we obtain the following bounds from Eqs. (2) and (3):

$$
f_{\mathrm{NL}}^{\text {enfold }}=148 \pm 118 \quad(68 \% \mathrm{CL}), \quad f_{\mathrm{NL}}^{\text {enfold }}=148 \pm 236 \quad(95 \% \mathrm{CL}) .
$$

For the local, orthogonal, and enfolded shapes the model with purely Gaussian perturbations $\left(f_{\mathrm{NL}}=0\right)$ is outside the $68 \%$ observational contour, but, apart from the orthogonal case, it is still consistent with the WMAP constraints at $95 \%$ CL.

In standard single-field inflation based on a canonical scalar field, Maldacena [12] showed that the non-linear parameter in the squeezed limit is given by $f_{\mathrm{NL}}^{\text {local }}=(5 / 12)\left(1-n_{\mathcal{R}}\right)$, where $n_{\mathcal{R}}$ is the scalar spectral index. Creminelli and Zaldarriaga [4] pointed out that the same non-Gaussianity consistency relation holds for any single-field model 
under the condition that only one mode of curvature perturbations survives after the Hubble radius crossing while the other one decays ${ }^{1}$ (see Refs. 50 53] for related works).

In the context of single-field k-inflation [54], the bispectrum of curvature perturbations was first derived by Seery and Lidsey in 2005 [18]. Since the scalar propagation speed squared $c_{s}^{2}$ can be much smaller than 1 [55], it is possible to realize the large equilateral non-linear parameter $\left|f_{\mathrm{NL}}^{\text {equil }}\right| \sim 1 / c_{s}^{2} \gg 1$. If we naively take the squeezed limit for the leading-order bispectrum derived in Refs. [18, 56], the term proportional to $1 / c_{s}^{2}$ does not disappear. This comes from the fact that the slow-variation corrections to the bispectrum need to be taken into account to estimate the local-type non-Gaussianity correctly. In fact, Chen et al. [56] showed that the Maldacena's consistency relation is recovered in the squeezed limit by carefully computing all the possible slow-variation corrections to the leading-order bispectrum. Thus the slow-variation single-field k-inflation models with $c_{s}^{2} \ll 1$ lead to small local non-Gaussianities, even though the equilateral non-linear parameter can be large.

In the Horndeski's most general scalar-tensor theories with second-order equations of motion [57 60], the leadingorder three-point correlation function of curvature perturbations was derived on the quasi de Sitter background [61, 62] (see Refs. 63 65] for the scalar non-Gaussianities in related Galileon models and Refs. [66] for the bispectrum of tensor perturbations in the Horndeski's theories). Although the result is valid for the estimation of the equilateral non-linear parameter, the bispectrum is not general enough to be used for any shape of non-Gaussianities. In this paper we take into account all the possible slow-variation corrections to the leading-order bispectrum in the Horndeski's theories ${ }^{2}$. Not only we reproduce the Maldacena's consistency relation in the squeezed limit, but we identify the shape close to the squeezed one at which the term $1 / c_{s}^{2}$ begins to contribute to the bispectrum.

Given our general expression of the bispectrum in the most general single-field scalar-tensor theories, we can evaluate the non-linear parameters of several different shapes to confront each inflationary model with observations. In particular the result $\left|f_{\mathrm{NL}}^{\text {local }}\right| \ll 1$ is robust for any slow-variation single-field model, so the detection of nonGaussianities in the squeezed limit will allow us to falsify the slow-variation single-field scenario. Note that in realistic observations the shape is not completely squeezed, in which case the bispectrum can be affected by the appearance of the term $1 / c_{s}^{2}$ mentioned above. Our results are useful to distinguish such difference accurately.

If $c_{s}^{2} \ll 1$, then the non-linear parameters $\left|f_{\mathrm{NL}}^{\text {equil }}\right|,\left|f_{\mathrm{NL}}^{\text {ortho }}\right|$, and $\left|f_{\mathrm{NL}}^{\text {enfold }}\right|$ can be much larger than the order of 1. Which shape dominates over the other ones depends on the models of inflation. In Ref. [62] it was shown that the correlation between the equilateral template and the shapes arising from the Horndeski's theories is quite high, but linear combinations of equilateral operators can give rise to a significantly different shape for a wide range of coefficients [44, 45]. In this regard we anticipate that there may be some models in which the shape orthogonal to the equilateral template provides an important contribution to the bispectrum.

In this paper we show that the leading-order three-point correlation function in the Horndeski's theories can be expressed by a linear combination of two bases whose shapes are highly correlated with equilateral and orthogonal shapes respectively. This decomposition is useful because the contributions from the equilateral and orthogonal shapes can be easily estimated for concrete models of inflation. We show that in k-inflation with the covariant Galileon terms there are cases in which the correlations with the orthogonal and enfolded templates are larger than that with the equilateral one. Thus the shapes of non-Gaussianities allow us to discriminate such models from observations.

This paper is organized as follows. In Sec. III we review the background and linear perturbation equations in the Horndeski's theories. In Sec. III we derive the three-point correlation function of curvature perturbations in the presence of slow-variation corrections to the leading-order bispectrum. In Sec. IV the non-linear parameter $f_{\mathrm{NL}}$ is evaluated in the squeezed, equilateral, and enfolded limits, respectively. In Sec. $\mathrm{V}$ we express the leading-order bispectrum in terms of equilateral and orthogonal bases. In Sec. VI we show concrete models of inflation in which the orthogonal and enfolded shapes can dominate over the equilateral one. Sec. VII is devoted to conclusions. In Appendix we show the details of the slow-variation corrections to the bispectrum.

\section{EQUATIONS OF MOTION FOR THE BACKGROUND AND LINEAR PERTURBATIONS}

The action corresponding to the most general scalar-tensor theories is given by [57, 58]

$$
\mathcal{S}=\int d^{4} x \sqrt{-g}\left[\frac{M_{\mathrm{pl}}^{2}}{2} R+P(\phi, X)-G_{3}(\phi, X) \square \phi+\mathcal{L}_{4}+\mathcal{L}_{5}\right]
$$

\footnotetext{
${ }^{1}$ If the decaying mode is non-negligible relative to the growing mode, the Maldacena's consistency relation can be violated 48, 49.

2 In the effective field theory of inflation (which allows the equations of motion higher than second order), a similar approach was taken by Cheung et al. [50] to show that the Maldacena's consistency relation holds in the squeezed limit. While the authors in this paper mainly focused on the local shape, we derive the full bispectrum in the Horndeski's theory which can be used for any shape of non-Gaussianities.
} 
where $g$ is the determinant of the metric $g_{\mu \nu}, M_{\mathrm{pl}}$ is the reduced Planck mass, $R$ is a Ricci scalar, and

$$
\begin{aligned}
& \mathcal{L}_{4}=G_{4}(\phi, X) R+G_{4, X}\left[(\square \phi)^{2}-\left(\nabla_{\mu} \nabla_{\nu} \phi\right)\left(\nabla^{\mu} \nabla^{\nu} \phi\right)\right], \\
& \mathcal{L}_{5}=G_{5}(\phi, X) G_{\mu \nu}\left(\nabla^{\mu} \nabla^{\nu} \phi\right)-\frac{1}{6} G_{5, X}\left[(\square \phi)^{3}-3(\square \phi)\left(\nabla_{\mu} \nabla_{\nu} \phi\right)\left(\nabla^{\mu} \nabla^{\nu} \phi\right)+2\left(\nabla^{\mu} \nabla_{\alpha} \phi\right)\left(\nabla^{\alpha} \nabla_{\beta} \phi\right)\left(\nabla^{\beta} \nabla_{\mu} \phi\right)\right] .(
\end{aligned}
$$

Here $P$ and $G_{i}$ 's $(i=3,4,5)$ are functions in terms of $\phi$ and $X=-\partial^{\mu} \phi \partial_{\mu} \phi / 2$ with the partial derivatives $G_{i, X} \equiv$ $\partial G_{i} / \partial X$, and $G_{\mu \nu}=R_{\mu \nu}-g_{\mu \nu} R / 2$ is the Einstein tensor ( $R_{\mu \nu}$ is the Ricci tensor).

We consider the following ADM metric [67] with scalar metric perturbations $\alpha, \psi$, and $\mathcal{R}$ about the flat FriedmannLemaître-Robertson-Walker (FLRW) background

$$
d s^{2}=-\left[(1+\alpha)^{2}-a(t)^{-2} e^{-2 \mathcal{R}}(\partial \psi)^{2}\right] d t^{2}+2 \partial_{i} \psi d t d x^{i}+a(t)^{2} e^{2 \mathcal{R}} d \boldsymbol{x}^{2},
$$

where $a(t)$ is the scale factor with cosmic time $t$. We choose the uniform field gauge $\delta \phi=0$, which fixes the timecomponent of a gauge-transformation vector $\xi^{\mu}$. The spatial part of $\xi^{\mu}$ is fixed by gauging away a perturbation $E$ that appears as a form $E_{, i j}$ in the metric (8).

The background equations of motion are given by

$$
\begin{aligned}
3 M_{\mathrm{pl}}^{2} H^{2} F+P+6 H G_{4, \phi} \dot{\phi}+\left(G_{3, \phi}-12 H^{2} G_{4, X}+9 H^{2} G_{5, \phi}-P_{, X}\right) \dot{\phi}^{2} & \\
+\left(6 G_{4, \phi X}-3 G_{3, X}-5 G_{5, X} H^{2}\right) & H \dot{\phi}^{3}+3\left(G_{5, \phi X}-2 G_{4, X X}\right) H^{2} \dot{\phi}^{4}-H^{3} G_{5, X X} \dot{\phi}^{5}=0 \\
\left(1-4 \delta_{G 4 X}-2 \delta_{G 5 X}+2 \delta_{G 5 \phi}\right) \epsilon= & \delta_{P X}+3 \delta_{G 3 X}-2 \delta_{G 3 \phi}+6 \delta_{G 4 X}-\delta_{G 4 \phi}-6 \delta_{G 5 \phi}+3 \delta_{G 5 X}+12 \delta_{G 4 X X}+2 \delta_{G 5 X X} \\
& -10 \delta_{G 4 \phi X}+2 \delta_{G 4 \phi \phi}-8 \delta_{G 5 \phi X}+2 \delta_{G 5 \phi \phi}-\delta_{\phi}\left(\delta_{G 3 X}+4 \delta_{G 4 X}-\delta_{G 4 \phi}\right. \\
& \left.+8 \delta_{G 4 X X}+3 \delta_{G 5 X}-4 \delta_{G 5 \phi}+2 \delta_{G 5 X X}-2 \delta_{G 4 \phi X}-4 \delta_{G 5 \phi X}\right),
\end{aligned}
$$

where $H=\dot{a} / a$ is the Hubble parameter (a dot represents a derivative with respect to $t$ ), $F=1+2 G_{4} / M_{\mathrm{pl}}^{2}$, and

$$
\begin{aligned}
& \epsilon=-\frac{\dot{H}}{H^{2}}, \quad \delta_{\phi}=\frac{\ddot{\phi}}{H \dot{\phi}}, \quad \delta_{P X}=\frac{P_{, X} X}{M_{\mathrm{pl}}^{2} H^{2} F}, \quad \delta_{G 3 X}=\frac{G_{3, X} \dot{\phi} X}{M_{\mathrm{pl}}^{2} H F}, \quad \delta_{G 3 \phi}=\frac{G_{3, \phi} X}{M_{\mathrm{pl}}^{2} H^{2} F}, \quad \delta_{G 4 X}=\frac{G_{4, X} X}{M_{\mathrm{pl}}^{2} F}, \\
& \delta_{G 4 \phi}=\frac{G_{4, \phi} \dot{\phi}}{M_{\mathrm{pl}}^{2} H F}, \quad \delta_{G 4 \phi X}=\frac{G_{4, \phi X} \dot{\phi} X}{M_{\mathrm{pl}}^{2} H F}, \quad \delta_{G 4 \phi \phi}=\frac{G_{4, \phi \phi} X}{M_{\mathrm{pl}}^{2} H^{2} F}, \quad \delta_{G 4 X X}=\frac{G_{4, X X} X^{2}}{M_{\mathrm{pl}}^{2} F}, \quad \delta_{G 5 \phi}=\frac{G_{5, \phi} X}{M_{\mathrm{pl}}^{2} F}, \\
& \delta_{G 5 X}=\frac{G_{5, X} H \dot{\phi} X}{M_{\mathrm{pl}}^{2} F}, \quad \delta_{G 5 X X}=\frac{G_{5, X X} H \dot{\phi} X^{2}}{M_{\mathrm{pl}}^{2} F} \quad \delta_{G 5 \phi X}=\frac{G_{5, \phi X} X^{2}}{M_{\mathrm{pl}}^{2} F}, \quad \delta_{G 5 \phi \phi}=\frac{G_{5, \phi \phi} \dot{\phi} X}{M_{\mathrm{pl}}^{2} H F},
\end{aligned}
$$

whose magnitudes are much smaller than 1 during inflation. The terms $\delta_{G 4 \phi X}, \delta_{G 4 \phi \phi}, \delta_{G 5 \phi X}, \delta G_{5 \phi \phi}$ as well as $\delta_{G 3 \phi X}=G_{3, \phi X} X^{2} /\left(M_{\mathrm{pl}}^{2} H^{2} F\right)$ and $\delta_{G 3 \phi \phi}=G_{3, \phi \phi} \dot{\phi} X /\left(M_{\mathrm{pl}}^{2} H^{3} F\right)$ are second-order of $\epsilon$. From Eq. (10) it follows that

$$
\epsilon=\delta_{P X}+3 \delta_{G 3 X}-2 \delta_{G 3 \phi}+6 \delta_{G 4 X}-\delta_{G 4 \phi}-6 \delta_{G 5 \phi}+3 \delta_{G 5 X}+12 \delta_{G 4 X X}+2 \delta_{G 5 X X}+\mathcal{O}\left(\epsilon^{2}\right)
$$

For the quantity $\delta_{F}=\dot{F} /(H F)$ we have

$$
\delta_{F}=2 \delta_{G 4 \phi}+\mathcal{O}\left(\epsilon^{2}\right)
$$

Using the relations between $\mathcal{R}, \psi$, and $\alpha$ that follows from Hamiltonian and momentum constraints, the second-order action for perturbations reduces to $[60,62,68]$

$$
\mathcal{S}_{2}=\int d t d^{3} x a^{3} Q\left[\dot{\mathcal{R}}^{2}-\frac{c_{s}^{2}}{a^{2}}(\partial \mathcal{R})^{2}\right]
$$

where

$$
\begin{aligned}
Q & =\frac{w_{1}\left(4 w_{1} w_{3}+9 w_{2}^{2}\right)}{3 w_{2}^{2}} \\
c_{s}^{2} & =\frac{3\left(2 w_{1}^{2} w_{2} H-w_{2}^{2} w_{4}+4 w_{1} \dot{w}_{1} w_{2}-2 w_{1}^{2} \dot{w}_{2}\right)}{w_{1}\left(4 w_{1} w_{3}+9 w_{2}^{2}\right)}
\end{aligned}
$$


and

$$
\begin{aligned}
w_{1}= & M_{\mathrm{pl}}^{2} F-4 X G_{4, X}-2 H X \dot{\phi} G_{5, X}+2 X G_{5, \phi}, \\
w_{2}= & 2 M_{\mathrm{pl}}^{2} H F-2 X \dot{\phi} G_{3, X}-16 H\left(X G_{4, X}+X^{2} G_{4, X X}\right)+2 \dot{\phi}\left(G_{4, \phi}+2 X G_{4, \phi X}\right) \\
& -2 H^{2} \dot{\phi}\left(5 X G_{5, X}+2 X^{2} G_{5, X X}\right)+4 H X\left(3 G_{5, \phi}+2 X G_{5, \phi X}\right), \\
w_{3}= & -9 M_{\mathrm{pl}}^{2} H^{2} F+3\left(X P_{, X}+2 X^{2} P_{, X X}\right)+18 H \dot{\phi}\left(2 X G_{3, X}+X^{2} G_{3, X X}\right)-6 X\left(G_{3, \phi}+X G_{3, \phi X}\right) \\
& +18 H^{2}\left(7 X G_{4, X}+16 X^{2} G_{4, X X}+4 X^{3} G_{4, X X X}\right)-18 H \dot{\phi}\left(G_{4, \phi}+5 X G_{4, \phi X}+2 X^{2} G_{4, \phi X X}\right) \\
& +6 H^{3} \dot{\phi}\left(15 X G_{5, X}+13 X^{2} G_{5, X X}+2 X^{3} G_{, 5 X X X}\right)-18 H^{2} X\left(6 G_{5, \phi}+9 X G_{5, \phi X}+2 X^{2} G_{5, \phi X X}\right), \\
w_{4}= & M_{\mathrm{pl}}^{2} F-2 X G_{5, \phi}-2 X G_{5, X} \ddot{\phi} .
\end{aligned}
$$

For later convenience we introduce the following parameter

$$
\epsilon_{s} \equiv \frac{Q c_{s}^{2}}{M_{\mathrm{pl}}^{2} F} \simeq \epsilon+\delta_{G 3 X}+\delta_{G 4 \phi}+8 \delta_{G 4 X X}+\delta_{G 5 X}+2 \delta_{G 5 X X}+\mathcal{O}\left(\epsilon^{2}\right) .
$$

At linear level the curvature perturbation obeys the equation of motion

$$
\left.\frac{\delta \mathcal{L}_{2}}{\delta \mathcal{R}}\right|_{1} \equiv-2\left[\frac{d}{d t}\left(a^{3} Q \dot{\mathcal{R}}\right)-a Q c_{s}^{2} \partial^{2} \mathcal{R}\right]=0
$$

We decompose $\mathcal{R}$ into the Fourier components, as

$$
\mathcal{R}(\tau, \boldsymbol{x})=\frac{1}{(2 \pi)^{3}} \int d^{3} k \mathcal{R}(\tau, \boldsymbol{k}) e^{i \boldsymbol{k} \cdot \boldsymbol{x}}, \quad \mathcal{R}(\tau, \boldsymbol{k})=u(\tau, \boldsymbol{k}) a(\boldsymbol{k})+u^{*}(\tau,-\boldsymbol{k}) a^{\dagger}(-\boldsymbol{k}),
$$

where $\tau=\int a^{-1} d t, \boldsymbol{k}$ is the comoving wave number, $a(\boldsymbol{k})$ and $a^{\dagger}(\boldsymbol{k})$ are the annihilation and creation operators, respectively, satisfying the commutation relations $\left[a\left(\boldsymbol{k}_{1}\right), a^{\dagger}\left(\boldsymbol{k}_{2}\right)\right]=(2 \pi)^{3} \delta^{(3)}\left(\boldsymbol{k}_{1}-\boldsymbol{k}_{2}\right)$ and $\left[a\left(\boldsymbol{k}_{1}\right), a\left(\boldsymbol{k}_{2}\right)\right]=$ $\left[a^{\dagger}\left(\boldsymbol{k}_{1}\right), a^{\dagger}\left(\boldsymbol{k}_{2}\right)\right]=0$.

Introducing a rescaled field $v=z u$ with $z=a \sqrt{2 Q}$, it follows that

$$
v^{\prime \prime}+\left(c_{s}^{2} k^{2}-\frac{z^{\prime \prime}}{z}\right) v=0
$$

where a prime represents a derivative with respect to $\tau$. Under the slow-variation approximation the term $z^{\prime \prime} / z$ can be expressed as

$$
\frac{z^{\prime \prime}}{z}=2(a H)^{2}\left(1-\frac{1}{2} \epsilon+\frac{3}{4} \eta_{s F}-\frac{3}{2} s\right)+\mathcal{O}\left(\epsilon^{2}\right)
$$

where

$$
\eta_{s F} \equiv \frac{\left(\epsilon_{s} F\right)^{\cdot}}{H\left(\epsilon_{s} F\right)}=\eta_{s}+\delta_{F}, \quad \eta_{s} \equiv \frac{\dot{\epsilon}_{s}}{H \epsilon_{s}}, \quad s \equiv \frac{\dot{c}_{s}}{H c_{s}} .
$$

Taking the dominant contribution in Eq. (25) and using the approximate relation $a \simeq-1 /(H \tau)$, we have $z^{\prime \prime} / z \simeq 2 / \tau^{2}$. The solution to Eq. (24), which recovers the Bunch-Davies vacuum state $\left(v=e^{-i c_{s} k \tau} / \sqrt{2 c_{s} k}\right)$ in the asymptotic past $(k \tau \rightarrow-\infty)$, is given by

$$
u(\tau, k)=\frac{i H e^{-i c_{s} k \tau}}{2\left(c_{s} k\right)^{3 / 2} \sqrt{Q}}\left(1+i c_{s} k \tau\right) .
$$

The slow-variation terms in Eq. (25) provide the corrections to the mode function (27). Later we shall discuss the effect of such corrections on the primordial non-Gaussianities.

The power spectrum $\mathcal{P}_{\mathcal{R}}\left(k_{1}\right)$ of curvature perturbations, some time after the Hubble radius crossing, is defined by $\left\langle 0\left|\mathcal{R}\left(0, \boldsymbol{k}_{1}\right) \mathcal{R}\left(0, \boldsymbol{k}_{2}\right)\right| 0\right\rangle=\left(2 \pi^{2} / k_{1}^{3}\right) \mathcal{P}_{\mathcal{R}}\left(k_{1}\right)(2 \pi)^{3} \delta^{(3)}\left(\boldsymbol{k}_{1}+\boldsymbol{k}_{2}\right)$. From Eq. (27) it follows that

$$
\mathcal{P}_{\mathcal{R}}=\frac{H^{2}}{8 \pi^{2} M_{\mathrm{pl}}^{2} \epsilon_{s} F c_{s}}
$$


which should be evaluated at $c_{s} k=a H$. The spectral index $n_{\mathcal{R}}$ is given by

$$
n_{\mathcal{R}}-\left.1 \equiv \frac{d \ln \mathcal{P}_{\mathcal{R}}}{d \ln k}\right|_{c_{s} k=a H}=-2 \epsilon-\eta_{s F}-s .
$$

The corrections to the solution (27) only give rise to the $\mathcal{O}\left(\epsilon^{2}\right)$ terms in Eq. (29).

Similarly the power spectrum $\mathcal{P}_{h}$ and the spectral index $n_{t}$ of gravitational waves are given, respectively, by [60, 62.

$$
\mathcal{P}_{h}=\frac{H^{2}}{2 \pi^{2} Q_{t} c_{t}^{3}} \simeq \frac{2 H^{2}}{\pi^{2} M_{\mathrm{pl}}^{2} F}, \quad n_{t}=\left.\frac{d \ln \mathcal{P}_{h}}{d \ln k}\right|_{c_{t} k=a H}=-2 \epsilon-\delta_{F},
$$

where $Q_{t}=w_{1} / 4=\left(M_{\mathrm{pl}}^{2} F / 4\right)\left(1-4 \delta_{G 4 X}-2 \delta_{G 5 X}+2 \delta_{G 5 \phi}\right)$ and $c_{t}^{2}=w_{4} / w_{1} \simeq 1+4 \delta_{G 4 X}+2 \delta_{G 5 X}-4 \delta_{G 5 \phi}$. When both $\mathcal{P}_{\mathcal{R}}$ and $\mathcal{P}_{h}$ remain constant, the tensor-to-scalar ratio can be evaluated as

$$
r=\frac{\mathcal{P}_{h}}{\mathcal{P}_{\mathcal{R}}} \simeq 16 c_{s} \epsilon_{s}
$$

\section{THREE-POINT CORRELATION FUNCTIONS IN THE PRESENCE OF CORRECTION TERMS}

In the Horndeski's theories the third-order action of perturbations was derived in Ref. [61, 62]. Here we do not repeat the details, but we summarize the main results. Under the approximation that all of the slow-variation terms in Eq. (11) are much smaller than 1, the third-order action reads

$$
\begin{aligned}
\mathcal{S}_{3}= & \int d t d^{3} x\left\{a^{3} \mathcal{C}_{1} M_{\mathrm{pl}}^{2} \mathcal{R} \dot{\mathcal{R}}^{2}+a \mathcal{C}_{2} M_{\mathrm{pl}}^{2} \mathcal{R}(\partial \mathcal{R})^{2}+a^{3} \mathcal{C}_{3} M_{\mathrm{pl}} \dot{\mathcal{R}}^{3}+a^{3} \mathcal{C}_{4} \dot{\mathcal{R}}\left(\partial_{i} \mathcal{R}\right)\left(\partial_{i} \mathcal{X}\right)+a^{3}\left(\mathcal{C}_{5} / M_{\mathrm{pl}}^{2}\right) \partial^{2} \mathcal{R}(\partial \mathcal{X})^{2}\right. \\
& +a \mathcal{C}_{6} \dot{\mathcal{R}}^{2} \partial^{2} \mathcal{R}+\mathcal{C}_{7}\left[\partial^{2} \mathcal{R}(\partial \mathcal{R})^{2}-\mathcal{R} \partial_{i} \partial_{j}\left(\partial_{i} \mathcal{R}\right)\left(\partial_{j} \mathcal{R}\right)\right] / a+a\left(\mathcal{C}_{8} / M_{\mathrm{pl}}\right)\left[\partial^{2} \mathcal{R} \partial_{i} \mathcal{R} \partial_{i} \mathcal{X}-\mathcal{R} \partial_{i} \partial_{j}\left(\partial_{i} \mathcal{R}\right)\left(\partial_{j} \mathcal{X}\right)\right] \\
& \left.+\left.\mathcal{F}_{1} \frac{\delta \mathcal{L}_{2}}{\delta \mathcal{R}}\right|_{1}\right\}
\end{aligned}
$$

where $\partial^{2} \mathcal{X}=Q \dot{\mathcal{R}}$. The dimensionless coefficients $\mathcal{C}_{i}(i=1, \cdots, 8)$ and the coefficient $\mathcal{F}_{1}$ are 62]

$$
\begin{aligned}
& \mathcal{C}_{1}=-\frac{3 F \epsilon_{s}}{c_{s}^{2}}\left(\frac{1}{c_{s}^{2}}-1\right)+\frac{F \epsilon_{s}}{c_{s}^{4}}\left(\epsilon_{s}-\eta_{s}-4 \delta_{G 3 X}-12 \delta_{G 4 X}-32 \delta_{G 4 X X}+12 \delta_{G 5 \phi}-10 \delta_{G 5 X}-8 \delta_{G 5 X X}\right)+\mathcal{O}\left(\epsilon^{3}\right), \\
& \mathcal{C}_{2}=F \epsilon_{s}\left(\frac{1}{c_{s}^{2}}-1\right)+\frac{F \epsilon_{s}}{c_{s}^{2}}\left(\epsilon_{s}+\eta_{s}-2 s+4 \delta_{G 4 X}+2 \delta_{G 5 X}-4 \delta_{G 5 \phi}\right)+\mathcal{O}\left(\epsilon^{3}\right), \\
& \mathcal{C}_{3}=\frac{F \epsilon_{s}}{c_{s}^{2}} \frac{M_{\mathrm{pl}}}{H}\left(\frac{1}{c_{s}^{2}}-1-\frac{2 \lambda}{\Sigma}\right)+\frac{F \epsilon_{s}}{c_{s}^{2}} \frac{M_{\mathrm{pl}}}{H}\left\{\frac{1}{c_{s}^{2}}\left(\delta_{G 3 X}+4 \delta_{G 4 X}+3 \delta_{G 5 X}-\delta_{G 4 \phi}-4 \delta_{G 5 \phi}+8 \delta_{G 4 X X}+2 \delta_{G 5 X X}\right)\right. \\
& -\left(3+2 \lambda_{3 X}\right) \delta_{G 3 X}-8\left(5+2 \lambda_{4 X}\right) \delta_{G 4 X X}-4\left(4+\lambda_{5 X}\right) \delta_{G 5 X X} \\
& \left.+\delta_{G 4 \phi}+8 \delta_{G 5 \phi}-8 \delta_{G 4 X}-9 \delta_{G 5 X}-6 \frac{c_{s}^{2}}{\epsilon_{s}}\left[\left(1+\lambda_{3 X}\right) \delta_{G 3 X}^{2}+\xi\left(\delta^{2}\right)\right]\right\}+\mathcal{O}\left(\epsilon^{3}\right), \\
& \mathcal{C}_{4}=-\frac{2 \epsilon_{s}}{c_{s}^{2}}+\mathcal{O}\left(\epsilon^{2}\right) \\
& \mathcal{C}_{5}=\frac{1}{4 F}\left(\epsilon_{s}-4 \delta_{G 3 X}-8 \delta_{G 4 X X}+8 \delta_{G 5 X}+4 \delta_{G 5 X X}\right)+\mathcal{O}\left(\epsilon^{2}\right), \\
& \mathcal{C}_{6}=2 F\left(\frac{M_{\mathrm{pl}}}{H}\right)^{2}\left[\left(1+\lambda_{3 X}\right) \delta_{G 3 X}+4\left(3+2 \lambda_{4 X}\right) \delta_{G 4 X X}+\delta_{G 5 X}+\left(5+2 \lambda_{5 X}\right) \delta_{G 5 X X}\right]+\mathcal{O}\left(\epsilon^{2}\right), \\
& \mathcal{C}_{7}=-\frac{2}{3} F\left(\frac{M_{\mathrm{pl}}}{H}\right)^{2}\left(\delta_{G 3 X}+6 \delta_{G 4 X X}+\delta_{G 5 X}+\delta_{G 5 X X}\right)+\mathcal{O}\left(\epsilon^{2}\right), \\
& \mathcal{C}_{8}=2 \frac{M_{\mathrm{pl}}}{H}\left(\delta_{G 3 X}+4 \delta_{G 4 X X}\right)+\mathcal{O}\left(\epsilon^{2}\right) \\
& \mathcal{F}_{1}=-\frac{L_{1} \mu_{1}+6 X \dot{\phi} G_{5, X}}{6 w_{1}^{2}}\left\{\left(\partial_{k} \mathcal{R}\right)\left(\partial_{k} \mathcal{X}\right)-\partial^{-2} \partial_{i} \partial_{j}\left[\left(\partial_{i} \mathcal{R}\right)\left(\partial_{j} \mathcal{X}\right)\right]\right\}-\frac{L_{1}}{c_{s}^{2}} \mathcal{R} \dot{\mathcal{R}} \\
& +\frac{L_{1}\left(L_{1} \mu_{1}+12 X \dot{\phi} G_{5, X}\right)}{12 w_{1} a^{2}}\left\{(\partial \mathcal{R})^{2}-\partial^{-2} \partial_{i} \partial_{j}\left[\left(\partial_{i} \mathcal{R}\right)\left(\partial_{j} \mathcal{R}\right)\right]\right\},
\end{aligned}
$$


where $\lambda_{3 X}=X G_{3, X X} / G_{3, X}, \lambda_{4 X}=X G_{4, X X X} / G_{4, X X}, \lambda_{5 X}=X G_{5, X X X} / G_{5, X X}, \mu_{1}=3 M_{\mathrm{pl}}^{2} F-24\left(X G_{4, X}+\right.$ $\left.X^{2} G_{4, X X}\right)-6 H \dot{\phi}\left(5 X G_{5, X}+2 X^{2} G_{5, X X}\right)+6 X\left(3 G_{5, \phi}+2 X G_{5, \phi X}\right), L_{1}=2 w_{1} / w_{2}$, and

$$
\begin{aligned}
\Sigma= & \frac{w_{1}\left(4 w_{1} w_{3}+9 w_{2}^{2}\right)}{12 M_{\mathrm{pl}}^{4}}, \\
\lambda= & \frac{F^{2}}{3}\left[3 X^{2} P_{, X X}+2 X^{3} P_{, X X X}+3 H \dot{\phi}\left(X G_{3, X}+5 X^{2} G_{3, X X}+2 X^{3} G_{3, X X X}\right)-2\left(2 X^{2} G_{3, \phi X}+X^{3} G_{3, \phi X X}\right)\right. \\
& +6 H^{2}\left(9 X^{2} G_{4, X X}+16 X^{3} G_{4, X X X}+4 X^{4} G_{4, X X X X}\right)-3 H \dot{\phi}\left(3 X G_{4 \phi, X}+12 X^{2} G_{4, \phi X X}+4 X^{3} G_{4, \phi X X X}\right) \\
& +H^{3} \dot{\phi}\left(3 X G_{5, X}+27 X^{2} G_{5, X X}+24 X^{3} G_{5, X X X}+4 X^{4} G_{5, X X X X}\right) \\
& \left.-6 H^{2}\left(6 X^{2} G_{5, \phi X}+9 X^{3} G_{5, \phi X X}+2 X^{4} G_{5, \phi X X X}\right)\right] .
\end{aligned}
$$

The explicit form of the second-order term $\xi\left(\delta^{2}\right)$ in Eq. [35] is given in Appendix of Ref. 62. The coefficient $\mathcal{F}_{1}$ involves the terms with the spatial and time derivatives of $\mathcal{R}$ and $\mathcal{X}$. These provide the corrections to the three-point correlation function higher than first order in slow-variation parameters ${ }^{3}$. Since we are interested in the bispectrum up to first order, we neglect the contribution of the term $\left.\mathcal{F}_{1}\left(\delta \mathcal{L}_{2} / \delta \mathcal{R}\right)\right|_{1}$ in the following discussion. We also evaluated other boundary terms and found that they only lead to the contribution higher than the order $\epsilon$.

The vacuum expectation value of $\mathcal{R}$ for the three-point operator in the asymptotic future $(\tau \rightarrow 0)$ is

$$
\left\langle\mathcal{R}\left(\boldsymbol{k}_{1}\right) \mathcal{R}\left(\boldsymbol{k}_{2}\right) \mathcal{R}\left(\boldsymbol{k}_{3}\right)\right\rangle=-i \int_{-\infty}^{0} d \tau a\left\langle 0\left|\left[\mathcal{R}\left(0, \boldsymbol{k}_{1}\right) \mathcal{R}\left(0, \boldsymbol{k}_{2}\right) \mathcal{R}\left(0, \boldsymbol{k}_{3}\right), \mathcal{H}_{\text {int }}(\tau)\right]\right| 0\right\rangle .
$$

The interacting Hamiltonian $\mathcal{H}_{\text {int }}$ is related to the third-order Lagrangian $\mathcal{L}_{3}$ as $\mathcal{H}_{\text {int }}=-\mathcal{L}_{3}$, where $\mathcal{S}_{3}=\int d t \mathcal{L}_{3}$. We write the three-point correlation function in the form

$$
\left\langle\mathcal{R}\left(\boldsymbol{k}_{1}\right) \mathcal{R}\left(\boldsymbol{k}_{2}\right) \mathcal{R}\left(\boldsymbol{k}_{3}\right)\right\rangle=(2 \pi)^{3} \delta^{(3)}\left(\boldsymbol{k}_{1}+\boldsymbol{k}_{2}+\boldsymbol{k}_{3}\right)\left(\mathcal{P}_{\mathcal{R}}\right)^{2} \mathcal{F}_{\mathcal{R}}\left(k_{1}, k_{2}, k_{3}\right),
$$

where

$$
\mathcal{F}_{\mathcal{R}}\left(k_{1}, k_{2}, k_{3}\right)=\frac{(2 \pi)^{4}}{\prod_{i=1}^{3} k_{i}^{3}} \mathcal{A}_{\mathcal{R}}\left(k_{1}, k_{2}, k_{3}\right) .
$$

If we use the leading-order solution (27) for the mode function and neglect the variation of the terms $\mathcal{C}_{i}$ 's for the integration of Eq. (44) with the approximation $a \simeq-1 /(H \tau)$, the resulting bispectrum is [61, 62]

$$
\begin{aligned}
\mathcal{A}_{\mathcal{R}} \supset & \frac{c_{s}^{2}}{4 \epsilon_{s} F} \mathcal{C}_{1} S_{1}+\frac{1}{4 \epsilon_{s} F} \mathcal{C}_{2} S_{2}+\frac{3 c_{s}^{2}}{2 \epsilon_{s} F} \frac{H}{M_{\mathrm{pl}}} \mathcal{C}_{3} S_{3}+\frac{1}{8} \mathcal{C}_{4} S_{4}+\frac{\epsilon_{s} F}{4 c_{s}^{2}} \mathcal{C}_{5} S_{5}+\frac{3}{\epsilon_{s} F}\left(\frac{H}{M_{\mathrm{pl}}}\right)^{2} \mathcal{C}_{6} S_{6} \\
& +\frac{1}{2 \epsilon_{s} F c_{s}^{2}}\left(\frac{H}{M_{\mathrm{pl}}}\right)^{2} \mathcal{C}_{7} S_{7}+\frac{1}{8 c_{s}^{2}} \frac{H}{M_{\mathrm{pl}}} \mathcal{C}_{8} S_{8},
\end{aligned}
$$

where

$$
\begin{aligned}
& S_{1}=\frac{2}{K} \sum_{i>j} k_{i}^{2} k_{j}^{2}-\frac{1}{K^{2}} \sum_{i \neq j} k_{i}^{2} k_{j}^{3}, \quad S_{2}=\frac{1}{2} \sum_{i} k_{i}^{3}+\frac{2}{K} \sum_{i>j} k_{i}^{2} k_{j}^{2}-\frac{1}{K^{2}} \sum_{i \neq j} k_{i}^{2} k_{j}^{3}, \quad S_{3}=\frac{\left(k_{1} k_{2} k_{3}\right)^{2}}{K^{3}}, \\
& S_{4}=\sum_{i} k_{i}^{3}-\frac{1}{2} \sum_{i \neq j} k_{i} k_{j}^{2}-\frac{2}{K^{2}} \sum_{i \neq j} k_{i}^{2} k_{j}^{3}, \quad S_{5}=\frac{1}{K^{2}}\left[\sum_{i} k_{i}^{5}+\frac{1}{2} \sum_{i \neq j} k_{i} k_{j}^{4}-\frac{3}{2} \sum_{i \neq j} k_{i}^{2} k_{j}^{3}-k_{1} k_{2} k_{3} \sum_{i>j} k_{i} k_{j}\right], \\
& S_{6}=S_{3}, \quad S_{7}=\frac{1}{K}\left(1+\frac{1}{K^{2}} \sum_{i>j} k_{i} k_{j}+\frac{3 k_{1} k_{2} k_{3}}{K^{3}}\right)\left[\frac{3}{4} \sum_{i} k_{i}^{4}-\frac{3}{2} \sum_{i>j} k_{i}^{2} k_{j}^{2}\right], \\
& S_{8}=\frac{1}{K^{2}}\left[\frac{3}{2} k_{1} k_{2} k_{3} \sum_{i} k_{i}^{2}-\frac{5}{2} k_{1} k_{2} k_{3} K^{2}-6 \sum_{i \neq j} k_{i}^{2} k_{j}^{3}-\sum_{i} k_{i}^{5}+\frac{7}{2} K \sum_{i} k_{i}^{4}\right]
\end{aligned}
$$

\footnotetext{
${ }^{3}$ Note that in Ref. [12] the term $\mathcal{R}^{2}$ is present in the expression of $\mathcal{F}_{1}$, which gives rise to the first-order contribution $\eta_{s}$. We absorb this term to other coefficients, so that the field definition in Ref. [12] is unnecessary.
} 
and $K=k_{1}+k_{2}+k_{3}$. The five shape functions $S_{i}$ 's $(i=1, \cdots, 5)$ are present in the context of k-inflation [18, 56]. In the Horndeski's theories the additional functions $S_{7}$ and $S_{8}$ appear, but they can be expressed by using other shape functions as 69 ]

$$
S_{7}=-\frac{3}{2}\left(3 S_{1}-S_{2}\right)+18 S_{3}, \quad S_{8}=3 S_{1}-S_{2}+3 S_{4} .
$$

Since the three functions $S_{6}, S_{7}$, and $S_{8}$ vanish in the limit $k_{3} \rightarrow 0$, the last three terms in Eq. (47) do not contribute to the local non-Gaussianities.

The bispectrum $\mathcal{A}_{\mathcal{R}}$ coming from the contributions of $\mathcal{C}_{1}, \mathcal{C}_{2}, \mathcal{C}_{3}, \mathcal{C}_{6}, \mathcal{C}_{7}$ are 0 -th order of $\epsilon$, while the bispectrum from $\mathcal{C}_{4}$ and $\mathcal{C}_{8}$ are first order. Since the term $\mathcal{C}_{5}$ leads to the bispectrum at the order of $\epsilon^{2}$, we can neglect its contribution. In the case where the leading-order terms of $\mathcal{A}_{\mathcal{R}}$ vanish (which occurs for local non-Gaussianities), we need to take into account next-order corrections to the bispectrum coming from the integrals that involve the terms $\mathcal{C}_{1}, \mathcal{C}_{2}, \mathcal{C}_{3}, \mathcal{C}_{6}, \mathcal{C}_{7}$ in Eq. (32). Using the linear equation of motion $\delta \mathcal{L}_{2} /\left.\delta \mathcal{R}\right|_{1}=0$, the $\mathcal{C}_{6}, \mathcal{C}_{7}$, and $\mathcal{C}_{8}$ dependent terms can be absorbed into the first five terms in Eq. (32) [69]. Then the third-order action (32) reads

$$
\mathcal{S}_{3}=\int d t d^{3} x\left\{a^{3} \tilde{\mathcal{C}}_{1} M_{\mathrm{pl}}^{2} \mathcal{R} \dot{\mathcal{R}}^{2}+a \tilde{\mathcal{C}}_{2} M_{\mathrm{pl}}^{2} \mathcal{R}(\partial \mathcal{R})^{2}+a^{3} \tilde{\mathcal{C}}_{3} M_{\mathrm{pl}} \dot{\mathcal{R}}^{3}+a^{3} \tilde{\mathcal{C}}_{4} \dot{\mathcal{R}}\left(\partial_{i} \mathcal{R}\right)\left(\partial_{i} \mathcal{X}\right)+a^{3}\left(\tilde{\mathcal{C}}_{5} / M_{\mathrm{pl}}^{2}\right) \partial^{2} \mathcal{R}(\partial \mathcal{X})^{2}\right\}
$$

The coefficients $\tilde{\mathcal{C}}_{i}(i=1, \cdots, 5)$, which give rise to the corrections up to the order of $\epsilon$ in $\mathcal{A}_{\mathcal{R}}$, are 69 ]

$$
\begin{aligned}
& \tilde{\mathcal{C}}_{1}=\mathcal{C}_{1}-\frac{3 H^{2}}{2 c_{s}^{4} M_{\mathrm{pl}}^{2}}\left(6+2 \epsilon+7 \eta_{s F}-5 \eta_{7}\right) \mathcal{C}_{7}+\frac{3 H \epsilon_{s} F}{2 c_{s}^{4} M_{\mathrm{pl}}} \mathcal{C}_{8} \\
& \tilde{\mathcal{C}}_{2}=\mathcal{C}_{2}+\frac{3 H^{2}}{2 c_{s}^{2} M_{\mathrm{pl}}^{2}}\left(2-2 \epsilon+\eta_{s}+\eta_{7}-4 s\right) \mathcal{C}_{7}-\frac{H \epsilon_{s} F}{2 c_{s}^{2} M_{\mathrm{pl}}} \mathcal{C}_{8}, \\
& \tilde{\mathcal{C}}_{3}=\mathcal{C}_{3}+\frac{H}{3 c_{s}^{2} M_{\mathrm{pl}}}\left(6+3 \eta_{s F}-4 s-\eta_{6}\right) \mathcal{C}_{6}+\frac{H}{c_{s}^{4} M_{\mathrm{pl}}}\left(6+3 \eta_{s F}-s-2 \eta_{7}\right) \mathcal{C}_{7}, \\
& \tilde{\mathcal{C}}_{4}=\mathcal{C}_{4}+\frac{3 H}{c_{s}^{2} M_{\mathrm{pl}}} \mathcal{C}_{8} \\
& \tilde{\mathcal{C}}_{5}=\mathcal{C}_{5}
\end{aligned}
$$

where $\eta_{i} \equiv \dot{\mathcal{C}}_{i} /\left(H \mathcal{C}_{i}\right)$, with $i=6,7,8$. The three-point correlation function analogous to (47) is given by

$$
\mathcal{A}_{\mathcal{R}} \supset \frac{c_{s}^{2}}{4 \epsilon_{s} F} \tilde{\mathcal{C}}_{1} S_{1}+\frac{1}{4 \epsilon_{s} F} \tilde{\mathcal{C}}_{2} S_{2}+\frac{3 c_{s}^{2}}{2 \epsilon_{s} F} \frac{H}{M_{\mathrm{pl}}} \tilde{\mathcal{C}}_{3} S_{3}+\frac{1}{8} \tilde{\mathcal{C}}_{4} S_{4}
$$

where we dropped the $\tilde{\mathcal{C}}_{5}$-dependent term. The difference between Eqs. (47) and (56) is that the bispectrum (56) includes the corrections coming from the time-variations of $\mathcal{C}_{6}$ and $\mathcal{C}_{7}$. However Eqs. (47) and (56) are equivalent at leading order. The terms $\tilde{\mathcal{C}}_{i}(i=1, \cdots, 4)$ can be expressed as

$$
\begin{aligned}
& \tilde{\mathcal{C}}_{1}=\tilde{\mathcal{C}}_{1}^{\text {lead }}+\frac{F}{c_{s}^{4}}\left[\epsilon_{s} \delta \mathcal{C}_{1}+\left(2 \epsilon+7 \eta_{s F}-5 \eta_{7}\right) \delta \mathcal{C}_{7}+3 \epsilon_{s} \delta \mathcal{C}_{8}\right] \\
& \tilde{\mathcal{C}}_{2}=\tilde{\mathcal{C}}_{2}^{\text {lead }}+\frac{F}{c_{s}^{2}}\left[\epsilon_{s} \delta \mathcal{C}_{2}+\left(2 \epsilon-\eta_{s F}-\eta_{7}+4 s\right) \delta \mathcal{C}_{7}-\epsilon_{s} \delta \mathcal{C}_{8}\right] \\
& \tilde{\mathcal{C}}_{3}=\tilde{\mathcal{C}}_{3}^{\text {lead }}+\frac{F M_{\mathrm{pl}}}{c_{s}^{2} H}\left[\epsilon_{s} \delta \mathcal{C}_{3}+\frac{2}{3}\left(3 \eta_{s F}-4 s-\eta_{6}\right) \delta \mathcal{C}_{6}-\frac{2}{3 c_{s}^{2}}\left(3 \eta_{s F}-s-2 \eta_{7}\right) \delta \mathcal{C}_{7}\right] \\
& \tilde{\mathcal{C}}_{4}=-\frac{2 \epsilon_{s}}{c_{s}^{2}}+\frac{6}{c_{s}^{2}} \delta \mathcal{C}_{8}
\end{aligned}
$$

where the leading-order terms are

$$
\begin{aligned}
& \tilde{\mathcal{C}}_{1}^{\text {lead }}=-\frac{3 F}{c_{s}^{2}}\left(\frac{1}{c_{s}^{2}}-1\right) \epsilon_{s}+\frac{6 F}{c_{s}^{4}} \delta \mathcal{C}_{7}, \\
& \tilde{\mathcal{C}}_{2}^{\text {lead }}=F\left(\frac{1}{c_{s}^{2}}-1\right) \epsilon_{s}-\frac{2 F}{c_{s}^{2}} \delta \mathcal{C}_{7}=-\frac{c_{s}^{2}}{3} \tilde{\mathcal{C}}_{1}^{\text {lead }}, \\
& \tilde{\mathcal{C}}_{3}^{\text {lead }}=\frac{F M_{\mathrm{pl}}}{c_{s}^{2} H}\left[\left(\frac{1}{c_{s}^{2}}-1-\frac{2 \lambda}{\Sigma}\right) \epsilon_{s}+4 \delta \mathcal{C}_{6}-\frac{4}{c_{s}^{2}} \delta \mathcal{C}_{7}\right],
\end{aligned}
$$


and $\delta \mathcal{C}_{i}$ 's are the first-order slow-variation terms given by

$$
\begin{aligned}
\delta \mathcal{C}_{1}= & \epsilon_{s}-\eta_{s}-4 \delta_{G 3 X}-12 \delta_{G 4 X}-32 \delta_{G 4 X X}+12 \delta_{G 5 \phi}-10 \delta_{G 5 X}-8 \delta_{G 5 X X} \\
\delta \mathcal{C}_{2}= & \epsilon_{s}+\eta_{s}-2 s+4 \delta_{G 4 X}+2 \delta_{G 5 X}-4 \delta_{G 5 \phi} \\
\delta \mathcal{C}_{3}= & \frac{1}{c_{s}^{2}}\left(\delta_{G 3 X}+4 \delta_{G 4 X}+3 \delta_{G 5 X}-\delta_{G 4 \phi}-4 \delta_{G 5 \phi}+8 \delta_{G 4 X X}+2 \delta_{G 5 X X}\right)-\left(3+2 \lambda_{3 X}\right) \delta_{G 3 X}-8\left(5+2 \lambda_{4 X}\right) \delta_{G 4 X X} \\
& -4\left(4+\lambda_{5 X}\right) \delta_{G 5 X X}+\delta_{G 4 \phi}+8 \delta_{G 5 \phi}-8 \delta_{G 4 X}-9 \delta_{G 5 X}-6 \frac{c_{s}^{2}}{\epsilon_{s}}\left[\left(1+\lambda_{3 X}\right) \delta_{G 3 X}^{2}+\xi\left(\delta^{2}\right)\right] \\
\delta \mathcal{C}_{6}= & \left(1+\lambda_{3 X}\right) \delta_{G 3 X}+4\left(3+2 \lambda_{4 X}\right) \delta_{G 4 X X}+\delta_{G 5 X}+\left(5+2 \lambda_{5 X}\right) \delta_{G 5 X X} \\
\delta \mathcal{C}_{7}= & \delta_{G 3 X}+6 \delta_{G 4 X X}+\delta_{G 5 X}+\delta_{G 5 X X} \\
\delta \mathcal{C}_{8}= & \delta_{G 3 X}+4 \delta_{G 4 X X}
\end{aligned}
$$

In order to derive the full expression of $\mathcal{A}_{\mathcal{R}}$ to the order of $\epsilon$, we need to compute the corrections to the first three integrations in Eq. (500). As studied in Ref. [56] in the context of k-inflation, there are several corrections to the bispectrum (56).

The first one comes from the variation of the coefficients $\tilde{\mathcal{C}}_{i}(i=1,2,3)$, i.e.,

$$
\tilde{\mathcal{C}}_{i}(\tau)=\tilde{\mathcal{C}}_{i}\left(\tau_{K}\right)-\frac{d \tilde{\mathcal{C}}_{i}}{d t} \frac{1}{H_{K}} \ln \frac{\tau}{\tau_{K}}+\mathcal{O}\left(\epsilon^{2} \tilde{\mathcal{C}}_{i}\right)
$$

We evaluate all the physical variables at the time $\tau_{K}=-1 /\left(K c_{s K}\right)$, which corresponds to the moment when the wave number $K=k_{1}+k_{2}+k_{3}$ crosses the Hubble radius $K c_{s K}=a_{K} H_{K}$.

The second one follows from the correction to the scale factor $a \simeq-1 /(H \tau)$, i.e.,

$$
a=-\frac{1}{H_{K} \tau}-\frac{\epsilon}{H_{K} \tau}+\frac{\epsilon}{H_{K} \tau} \ln \left(\tau / \tau_{K}\right)+\mathcal{O}\left(\epsilon^{2}\right) .
$$

Thirdly, the mode function (27) is subject to change by taking into account the $\mathcal{O}(\epsilon)$ terms on the r.h.s. of Eq. (25) :

$$
u(y)=-\frac{\sqrt{\pi}}{2 \sqrt{2}} \frac{1}{\sqrt{\epsilon_{s} F c_{s}}} \frac{H}{M_{\mathrm{pl}}} \frac{y^{3 / 2}}{k^{3 / 2}}\left(1+\frac{1}{2} \epsilon+\frac{1}{2} s\right) e^{i \frac{\pi}{2}\left(\epsilon+\frac{1}{2} \eta_{s F}\right)} H_{\nu}^{(1)}[(1+\epsilon+s) y],
$$

where $y=c_{s} k /(a H), \nu=3 / 2+\epsilon+\eta_{s F} / 2+s / 2$, and $H_{\nu}^{(1)}(x)$ is the Hankel function of the first kind. In the large-scale limit $(y \rightarrow 0)$ the Hankel function behaves as $H_{\nu}^{(1)}(x) \rightarrow-i /[\sin (\pi \nu) \Gamma(1-\nu)](x / 2)^{-\nu}$ and hence the mode function approaches

$$
u(0)=\frac{i}{2 k^{3 / 2}} \frac{1}{\sqrt{\left(\epsilon_{s} F\right)_{k} c_{s k}}} \frac{H_{k}}{M_{\mathrm{pl}}} \frac{1}{k^{3 / 2}}\left[1-\left(\gamma_{2}+1\right) \epsilon-\frac{\gamma_{2}}{2} \eta_{s F}-\left(\frac{\gamma_{2}}{2}+1\right) s\right] e^{i \frac{\pi}{2}\left(\epsilon+\frac{1}{2} \eta_{s F}\right)},
$$

where $\gamma_{2}=\gamma_{1}-2+\ln 2 \simeq-0.7296 \ldots$ and $\gamma_{1}=0.5772 \ldots$ is the Euler-Mascheroni constant. Note that the quantities with the subscript $k$ in Eq. (73) are evaluated at $c_{s k} k /\left(a_{k} H_{k}\right)=1$. For the wave number $k_{i}$ there is the running from $k_{i}$ to $K$, as

$$
\frac{1}{\sqrt{\left(\epsilon_{s} F\right)_{k_{i}} c_{s k_{i}}}} \frac{H_{k_{i}}}{M_{\mathrm{pl}}}=\frac{1}{\sqrt{\left(\epsilon_{s} F\right)_{K} c_{s K}}} \frac{H_{K}}{M_{\mathrm{pl}}}\left[1-\left(\epsilon+\frac{1}{2} \eta_{s F}+\frac{1}{2} s\right) \ln \frac{k_{i}}{K}\right]+\mathcal{O}\left(\epsilon^{2}\right) .
$$

Writing the correction to the leading order solution (27) as $\Delta u^{*}(\tau, k)$, it follows that

$$
\begin{aligned}
& \Delta u^{*}\left(\tau, k_{i}\right)=-\frac{1}{2 k_{i}^{3 / 2} \sqrt{\left(\epsilon_{s} F\right)_{K} c_{s K}}} \frac{H_{K}}{M_{\mathrm{pl}}} e^{-i \frac{\pi}{2}\left(\epsilon+\frac{1}{2} \eta_{s F}\right)} e^{-i x}\left[(\epsilon+s)(x-i)+i s x^{2}\right. \\
& \left.+\left\{\left(\epsilon+\frac{1}{2} \eta_{s F}+\frac{1}{2} s\right)(i-x)-i s x^{2}\right\} \ln \frac{\tau}{\tau_{K}}+\sqrt{\frac{\pi}{2}} e^{i x}\left(\epsilon+\frac{1}{2} \eta_{s F}+\frac{1}{2} s\right) x^{3 / 2}\left(\frac{d H_{\nu}^{(1) *}}{d \nu}\right)_{\nu=3 / 2}\right], \\
& \frac{d}{d \tau} \Delta u^{*}\left(\tau, k_{i}\right)=\frac{1}{2 k_{i}^{3 / 2} \sqrt{\left(\epsilon_{s} F\right)_{K} c_{s K}}} \frac{H_{K}}{M_{\mathrm{pl}}} e^{-i \frac{\pi}{2}\left(\epsilon+\frac{1}{2} \eta_{s F}\right)} k_{i} c_{s K} e^{-i x}\left[x(s x-i \epsilon)+\left(\epsilon+\frac{1}{2} \eta_{s F}+\frac{1}{2} s\right)\left(\frac{i}{x}-1\right)\right. \\
& \left.+i\left(\epsilon+\frac{1}{2} \eta_{s F}-\frac{3}{2} s+i s x\right) x \ln \frac{\tau}{\tau_{K}}+\sqrt{\frac{\pi}{2}} e^{i x}\left(\epsilon+\frac{1}{2} \eta_{s F}+\frac{1}{2} s\right) \frac{d}{d x}\left(x^{3 / 2}\left(\frac{d H_{\nu}^{(1) *}}{d \nu}\right)_{\nu=3 / 2}\right)\right],
\end{aligned}
$$


where $x \equiv-k_{i} c_{s K} \tau$.

In Appendix A we give the explicit forms of corrections to the first three terms in Eq. (56). Each correction can be expressed as

$$
\Delta \mathcal{A}_{\mathcal{R}}^{(1)}=\left(\frac{c_{s}^{2}}{4 \epsilon_{s} F} \tilde{\mathcal{C}}_{1}^{\text {lead }}\right)_{K} \delta Q_{1}, \quad \Delta \mathcal{A}_{\mathcal{R}}^{(2)}=-\left(\frac{1}{4 \epsilon_{s} F} \tilde{\mathcal{C}}_{2}^{\text {lead }}\right)_{K} \delta Q_{2}, \quad \Delta \mathcal{A}_{\mathcal{R}}^{(3)}=\left(\frac{3 c_{s}^{2} H}{4 \epsilon_{s} F M_{\mathrm{pl}}} \tilde{\mathcal{C}}_{3}^{\text {lead }}\right)_{K} \delta Q_{3},
$$

where $\delta Q_{1}, \delta Q_{2}$, and $\delta Q_{3}$ are the $\mathcal{O}(\epsilon)$ terms derived by summing up the contributions (A1)-A5), (A6)-A And (A10)- (A13), respectively. On using Eqs. 61)- 663), it follows that

$$
\begin{aligned}
\Delta \mathcal{A}_{\mathcal{R}} & =\Delta \mathcal{A}_{\mathcal{R}}^{(1)}+\Delta \mathcal{A}_{\mathcal{R}}^{(2)}+\Delta \mathcal{A}_{\mathcal{R}}^{(3)} \\
& =-\frac{1}{4}\left(\frac{1}{c_{s}^{2}}-1-\frac{2}{c_{s}^{2}} \frac{\delta \mathcal{C}_{7}}{\epsilon_{s}}\right)\left(3 \delta Q_{1}+\delta Q_{2}\right)+\frac{3}{4}\left(\frac{1}{c_{s}^{2}}-1-\frac{2 \lambda}{\Sigma}+4 \frac{\delta \mathcal{C}_{6}}{\epsilon_{s}}-\frac{4}{c_{s}^{2}} \frac{\delta \mathcal{C}_{7}}{\epsilon_{s}}\right) \delta Q_{3} .
\end{aligned}
$$

The explicit forms of $3 \delta Q_{1}+\delta Q_{2}$ and $\delta Q_{3}$ are

$$
\begin{aligned}
3 \delta & Q_{1}+\delta Q_{2} \\
= & {\left[-2\left(7+2 \gamma_{1}+6 \gamma_{2}\right) \epsilon+3\left(1-2 \gamma_{1}-2 \gamma_{2}\right) \eta_{s F}-\left(21-14 \gamma_{1}+6 \gamma_{2}\right) s-2\left(1-2 \gamma_{1}\right) \tilde{\eta}_{1}-2\left(2 \epsilon+\eta_{s F}+s\right) \ln \frac{k_{1} k_{2} k_{3}}{K^{3}}\right] } \\
& \times \frac{1}{K} \sum_{i>j} k_{i}^{2} k_{j}^{2}+\left[2\left(3+\gamma_{1}+3 \gamma_{2}\right) \epsilon-3\left(1-\gamma_{1}-\gamma_{2}\right) \eta_{s F}+\left(17-\frac{35}{2} \gamma_{1}+3 \gamma_{2}\right) s+2\left(1-\gamma_{1}\right) \tilde{\eta}_{1}\right. \\
& \left.+\left(2 \epsilon+\eta_{s F}+s\right) \ln \frac{k_{1} k_{2} k_{3}}{K^{3}}\right] \frac{1}{K^{2}} \sum_{i \neq j} k_{i}^{2} k_{j}^{3}+3\left(2 \gamma_{1}-1\right) s\left[\frac{1}{K^{3}} \sum_{i \neq j} k_{i}^{2} k_{j}^{4}+\frac{2}{K^{3}} \sum_{i>j} k_{i}^{3} k_{j}^{3}-3 \frac{\left(k_{1} k_{2} k_{3}\right)^{2}}{K^{3}}\right] \\
& -\frac{1}{4}\left[22 \epsilon+9 \eta_{s F}+2 \tilde{\eta}_{1}+\left(11+4 \gamma_{1}\right) s\right] k_{1} k_{2} k_{3}-\left(\frac{1}{2} \epsilon+\frac{3}{4} \eta_{s F}-\frac{1}{2} \tilde{\eta}_{1}-\frac{1}{4} s\right) \sum_{i \neq j} k_{i} k_{j}^{2} \\
& +\left[\frac{1}{2}\left(3+\gamma_{1}+3 \gamma_{2}\right) \epsilon-\frac{3}{4}\left(1-\gamma_{1}-\gamma_{2}\right) \eta_{s F}+\frac{1}{2}\left(1-\gamma_{1}\right) \tilde{\eta}_{1}+\frac{1}{4}\left(13-\gamma_{1}+3 \gamma_{2}\right) s+\frac{1}{4}\left(2 \epsilon+\eta_{s F}+s\right) \ln \frac{k_{1} k_{2} k_{3}}{K^{3}}\right] \sum_{i} k_{i}^{3} \\
& -\frac{1}{2}\left(1+\gamma_{1}\right) s \frac{1}{K} \sum_{i} k_{i}^{4}-\frac{1}{2} \gamma_{1} s \frac{1}{K^{2}} \sum_{i \neq j} k_{i} k_{j}^{4}+\frac{3}{2}\left(2 \epsilon+\eta_{s F}+s\right)\left(2 \sum k_{i}^{3}+2 \sum_{i \neq j} k_{i} k_{j}^{2}-\mathcal{V}\right), \\
\delta Q_{3} & {\left[-2\left(2+2 \gamma_{1}+3 \gamma_{2}\right) \epsilon+3\left(\frac{3}{2}-\gamma_{1}-\gamma_{2}\right) \eta_{s F}+\left(3 \gamma_{1}-3 \gamma_{2}-\frac{29}{2}\right) s+\left(2 \gamma_{1}-3\right) \tilde{\eta}_{3}-\left(2 \epsilon+\eta_{s F}+s\right) \ln \frac{k_{1} k_{2} k_{3}}{K^{3}}\right] } \\
& \times \frac{\left(k_{1} k_{2} k_{3}\right)^{2}}{K^{3}}+\left(\epsilon+\frac{1}{2} \eta_{s F}+\frac{1}{2} s\right)\left(\frac{1}{K^{2}} \sum_{i \neq j} k_{i}^{2} k_{j}^{3}-\frac{2}{K} \sum_{i>j} k_{i}^{2} k_{j}^{2}+\mathcal{U}\right),
\end{aligned}
$$

where $\tilde{\eta}_{i}=\left(d \tilde{\mathcal{C}}_{i}^{\text {lead }} / d t\right) /\left(H \tilde{\mathcal{C}}_{i}^{\text {lead }}\right)$, and

$$
\begin{aligned}
\mathcal{V} \equiv & \mathcal{M}-\left(k_{2}^{3}+k_{3}^{3}\right) \operatorname{Re}\left[\int_{0}^{\infty} d x_{1} \frac{e^{-i K x_{1} / k_{1}}}{x_{1}}\right]-\frac{k_{2}^{2} k_{3}^{2}}{k_{1}} \mathcal{G}-\frac{1}{3} \mathcal{N}+\text { perm. } \\
= & -k_{1} \operatorname{Re}\left[\int_{0}^{\infty} d x_{1} \frac{1}{x_{1}}\left(k_{2}^{2}+k_{3}^{2}+i k_{2} k_{3} \frac{k_{2}+k_{3}}{k_{1}} x_{1}\right) e^{-i \frac{k_{2}+k_{3}}{k_{1}} x_{1}} \frac{d h^{*}\left(x_{1}\right)}{d x_{1}}\right]-\left(k_{2}^{3}+k_{3}^{3}\right) \operatorname{Re}\left[\int_{0}^{\infty} d x_{1} \frac{e^{-i K x_{1} / k_{1}}}{x_{1}}\right] \\
& +\frac{k_{1}}{6} \sum_{i} k_{i}^{2} \operatorname{Re}\left[\int_{0}^{\infty} d x_{1} \frac{1}{x_{1}^{2}} e^{-i x_{1} \frac{k_{2}+k_{3}}{k_{1}}}\left(1+i \frac{k_{2}+k_{3}}{k_{1}} x_{1}-\frac{k_{2} k_{3}}{k_{1}^{2}} x_{1}^{2}\right) h^{*}\left(x_{1}\right)\right] \\
& -\frac{k_{2}^{2} k_{3}^{2}}{k_{1}} \operatorname{Re}\left[\int_{0}^{\infty} d x_{1} h^{*}\left(x_{1}\right) e^{-i \frac{k_{2}+k_{3}}{k_{1}} x_{1}}\right]+\text { perm. } \\
\mathcal{U} \equiv & \frac{k_{2}^{2} k_{3}^{2}}{k_{1}} \operatorname{Re}\left[\int_{0}^{\infty} d x_{1} h^{*}\left(x_{1}\right)\left(1-i \frac{k_{2}+k_{3}}{k_{1}} x_{1}\right) e^{-i \frac{k_{2}+k_{3}}{k_{1}} x_{1}}\right]+\operatorname{perm.} \\
h(x) \equiv & \sqrt{\frac{\pi}{2}} x^{3 / 2}\left[\frac{d H_{\nu}^{(1)}(x)}{d \nu}\right]_{\nu=3 / 2}=-2 i e^{i x}+i e^{-i x}(1+i x)[\operatorname{Ci}(2 x)+i \operatorname{Si}(2 x)]-i \pi \sin x+i \pi x \cos x
\end{aligned}
$$


The definition of $\mathcal{M}, \mathcal{G}$, and $\mathcal{N}$ is given in Appendix A. In Eq. (81), we have used the relation between the variables $x_{K}=-K c_{s K} \tau$ and $x_{i}=-k_{i} c_{s K} \tau(i=1,2,3)$, as $x_{K}=\left(K / k_{i}\right) x_{i}$ (without summation over $i$ ). The variable $x_{K}=-K c_{s K} \tau$ is related to $x_{i}=-k_{i} c_{s K} \tau(i=1,2,3)$, as $x_{K}=\left(K / k_{i}\right) x_{i}$. The symbol "perm." stands for cyclic permutations with respect to $k_{1}, k_{2}$, and $k_{3}$. In Eq. (79) we also used the relation $\tilde{\eta}_{2}=\tilde{\eta}_{1}+2 s$ to eliminate $\tilde{\eta}_{2}$ (which follows from $\left.\tilde{\mathcal{C}}_{2}^{\text {lead }}=-\left(c_{s}^{2} / 3\right) \tilde{\mathcal{C}}_{1}^{\text {lead }}\right)$. In Appendix B we evaluate the values of $\mathcal{V}$ and $\mathcal{U}$ as functions of $r_{2} \equiv k_{2} / k_{1}$ and $r_{3} \equiv k_{3} / k_{1}$.

The total bispectrum $\mathcal{A}_{\mathcal{R}}$ is the sum of Eqs. (56) and (78), which can be written as

$$
\mathcal{A}_{\mathcal{R}}=\mathcal{A}_{\mathcal{R}}^{\text {lead }}+\mathcal{A}_{\mathcal{R}}^{\text {corre }}
$$

where

$$
\begin{aligned}
\mathcal{A}_{\mathcal{R}}^{\text {lead }}= & {\left[\frac{1}{4}\left(1-\frac{1}{c_{s}^{2}}\right)+\frac{1}{2 c_{s}^{2}} \frac{\delta \mathcal{C}_{7}}{\epsilon_{s}}\right]\left(3 S_{1}-S_{2}\right)+\left[\frac{3}{2}\left(\frac{1}{c_{s}^{2}}-1\right)-\frac{3 \lambda}{\Sigma}+\frac{6 \delta \mathcal{C}_{6}}{\epsilon_{s}}-\frac{6}{c_{s}^{2}} \frac{\delta \mathcal{C}_{7}}{\epsilon_{s}}\right] S_{3}, } \\
\mathcal{A}_{\mathcal{R}}^{\text {corre }}= & \frac{1}{4 c_{s}^{2}}\left[\delta \mathcal{C}_{1}+\left(2 \epsilon+7 \eta_{s F}-5 \eta_{7}\right) \frac{\delta \mathcal{C}_{7}}{\epsilon_{s}}+3 \delta \mathcal{C}_{8}\right] S_{1}+\frac{1}{4 c_{s}^{2}}\left[\delta \mathcal{C}_{2}+\left(2 \epsilon-\eta_{s F}-\eta_{7}+4 s\right) \frac{\delta \mathcal{C}_{7}}{\epsilon_{s}}-\delta \mathcal{C}_{8}\right] S_{2} \\
& +\left[\frac{3}{2} \delta \mathcal{C}_{3}+\left(3 \eta_{s F}-\eta_{6}-4 s\right) \frac{\delta \mathcal{C}_{6}}{\epsilon_{s}}-\frac{1}{c_{s}^{2}}\left(3 \eta_{s}-2 \eta_{7}-s\right) \frac{\delta \mathcal{C}_{7}}{\epsilon_{s}}\right] S_{3}-\frac{1}{4 c_{s}^{2}}\left(\epsilon_{s}-3 \delta \mathcal{C}_{8}\right) S_{4} \\
& -\frac{1}{4}\left(\frac{1}{c_{s}^{2}}-1-\frac{2}{c_{s}^{2}} \frac{\delta \mathcal{C}_{7}}{\epsilon_{s}}\right)\left(3 \delta Q_{1}+\delta Q_{2}\right)+\frac{3}{4}\left(\frac{1}{c_{s}^{2}}-1-\frac{2 \lambda}{\Sigma}+4 \frac{\delta \mathcal{C}_{6}}{\epsilon_{s}}-\frac{4}{c_{s}^{2}} \frac{\delta \mathcal{C}_{7}}{\epsilon_{s}}\right) \delta Q_{3} .
\end{aligned}
$$

The leading-order bispectrum $\mathcal{A}_{\mathcal{R}}^{\text {lead }}$ (given already in Refs. [61, 62]) and the correction $\mathcal{A}_{\mathcal{R}}^{\text {corre }}$ are of the orders of $\mathcal{O}\left(\epsilon^{0}\right)$ and $\mathcal{O}(\epsilon)$, respectively.

\section{LOCAL, EQUILATERAL, AND ENFOLDED NON-GAUSSIANITIES}

The non-linear parameter characterizing the strength of non-Gaussianities is defined by

$$
f_{\mathrm{NL}}=\frac{10}{3} \frac{\mathcal{A}_{\mathcal{R}}}{\sum_{i=1}^{3} k_{i}^{3}} .
$$

In the following we estimate $f_{\mathrm{NL}}$ for three different shapes of non-Gaussianities.

\section{A. Local non-Gaussianities}

The local shape corresponds to $k_{3} \rightarrow 0$ and $k_{2} \rightarrow k_{1} \equiv k$, in which case $f_{\mathrm{NL}}^{\text {local }}=(5 / 3) \mathcal{A}_{\mathcal{R}} / k^{3}$. Since $S_{1}=k^{3} / 2$, $S_{2}=3 k^{3} / 2=3 S_{1}$, and $S_{3}=S_{4}=0$, the leading-order bispectrum (85) vanishes. In the limit that $k_{3} \rightarrow 0$ the function $\mathcal{U}$ given by Eq. (82) approaches $k^{3} / 2$ [56] (see also Appendix B), so that the term $\delta Q_{3}$ in Eq. (80) vanishes. Then the bispectrum (86) reduces

$$
\mathcal{A}_{\mathcal{R}}^{\text {corre }}=\frac{k^{3}}{8 c_{s}^{2}}\left(\delta \mathcal{C}_{1}+3 \delta \mathcal{C}_{2}\right)-\frac{1}{4}\left(\frac{1}{c_{s}^{2}}-1\right)\left(3 \delta Q_{1}+\delta Q_{2}\right)+\frac{\delta \mathcal{C}_{7}}{8 c_{s}^{2} \epsilon_{s}}\left[4\left(2 \epsilon+\eta_{s F}-2 \eta_{7}+3 s\right) k^{3}+4\left(3 \delta Q_{1}+\delta Q_{2}\right)\right] .
$$

Using Eqs. (64) and (65) together with the relations (13), (21), and (26), we have $\delta \mathcal{C}_{1}+3 \delta \mathcal{C}_{2}=4 \epsilon+2 \eta_{s F}-6 s$. In the limit $k_{3} \rightarrow 0$ the function $\mathcal{V}$ behaves as $\mathcal{V} \rightarrow 20 k^{3} / 3$ [56] (see also Appendix B), so that Eq. (79) reduces to $3 \delta Q_{1}+\delta Q_{2} \rightarrow\left(2 \epsilon-\eta_{s F}+5 s+2 \tilde{\eta}_{1}\right) k^{3}$. Then Eq. (88) reads

$$
\mathcal{A}_{\mathcal{R}}^{\text {corre }}=\frac{k^{3}}{4}\left(2 \epsilon-\eta_{s F}+5 s+2 \tilde{\eta}_{1}\right)+\frac{k^{3}}{2 c_{s}^{2}}\left(\eta_{s F}-4 s-\tilde{\eta}_{1}\right)+k^{3} \frac{\delta \mathcal{C}_{7}}{c_{s}^{2} \epsilon_{s}}\left(2 \epsilon+4 s+\tilde{\eta}_{1}-\eta_{7}\right) .
$$

Taking the time-derivatives of $\tilde{\mathcal{C}}_{1}^{\text {lead }}$ and $\mathcal{C}_{7}$, we obtain the following relation

$$
\frac{\delta \mathcal{C}_{7}}{c_{s}^{2} \epsilon_{s}}\left(2 \epsilon+4 s+\tilde{\eta}_{1}-\eta_{7}\right)=\frac{1}{2}\left(\eta_{s F}-\tilde{\eta}_{1}-2 s\right)-\frac{1}{2 c_{s}^{2}}\left(\eta_{s F}-4 s-\tilde{\eta}_{1}\right) .
$$

Substituting Eq. (90) into Eq. (89), it follows that

$$
\mathcal{A}_{\mathcal{R}}^{\text {corre }}=\frac{k^{3}}{4}\left(2 \epsilon+\eta_{s F}+s\right) \text {. }
$$


Using the spectral index $n_{\mathcal{R}}$ given in Eq. (29), the non-linear parameter is expressed as

$$
f_{\mathrm{NL}}^{\text {local }}=\frac{5}{12}\left(1-n_{\mathcal{R}}\right) .
$$

This matches with the Maldacena's result [12] derived for a canonical scalar field (see also Refs. [50, 53, 56] for the derivation of the same relation in other single field models). Creminelli and Zaldarriaga [47] pointed out that the consistency relation (92) should hold for any slow-variation single-field inflation. In fact we have shown that this holds for most general single-field scalar-tensor theories with second-order equations of motion by explicitly computing the slow-variation corrections to the bispectrum (56). Since $\left|f_{\mathrm{NL}}^{\text {local }}\right|$ is much smaller than 1 in such models, the observational detection of local non-Gaussianities with $\left|f_{\mathrm{NL}}^{\text {local }}\right| \gtrsim 1$ implies that we need to go beyond the slow-variation single-field scenario.

In the limit that $k_{3} \rightarrow 0$ the shape functions $S_{6}, S_{7}$, and $S_{8}$ vanish. This means that the functions $\mathcal{C}_{6}, \mathcal{C}_{7}, \mathcal{C}_{8}$ on the r.h.s. of Eqs. (51)-(54) do not contribute to the local non-Gaussianities. In fact, we can derive the consistency relation (92) by setting $\delta \mathcal{C}_{7}=0$ in Eqs. (88) and (90). In this sense the situation is analogous to that in k-inflation.

Let us consider the not-so squeezed case in which the ratio $r_{3}=k_{3} / k_{1}$ is non-vanishing, i.e., $0<r_{3} \ll 1$ and $k_{1}=k_{2}$. The leading-order non-linear parameter following from Eq. (85) is given by

$$
f_{\mathrm{NL}}^{\text {lead }}=\frac{5 r_{3}^{2}\left[\alpha_{1}\left(22+4 r_{3}-4 r_{3}^{2}-r_{3}^{3}\right)\left(2+r_{3}\right)+2 \alpha_{2}\right]}{3\left(2+r_{3}\right)^{3}\left(2+r_{3}^{3}\right)}
$$

where

$$
\alpha_{1}=\frac{1}{4}\left(1-\frac{1}{c_{s}^{2}}\right)+\frac{1}{2 c_{s}^{2}} \frac{\delta \mathcal{C}_{7}}{\epsilon_{s}}, \quad \alpha_{2}=\frac{3}{2}\left(\frac{1}{c_{s}^{2}}-1\right)-\frac{3 \lambda}{\Sigma}+\frac{6 \delta \mathcal{C}_{6}}{\epsilon_{s}}-\frac{6}{c_{s}^{2}} \frac{\delta \mathcal{C}_{7}}{\epsilon_{s}}
$$

In the regime $r_{3} \ll 1$ we have $f_{\mathrm{NL}}^{\text {lead }} \simeq 5\left(22 \alpha_{1}+\alpha_{2}\right) r_{3}^{2} / 24$. The values of $\alpha_{1}$ and $\alpha_{2}$ depend on the models, but for $c_{s}^{2} \ll 1$ they are at most of the order of $1 / c_{s}^{2}$. In this case the leading-order non-linear parameter can be estimated as

$$
\left|f_{\mathrm{NL}}^{\text {lead }}\right| \approx \frac{r_{3}^{2}}{c_{s}^{2}}
$$

Then the transition from the value (92) to the value (95) occurs at

$$
r_{3} \approx c_{s} \sqrt{1-n_{\mathcal{R}}}
$$

The effect of the term $1 / c_{s}^{2}$ in Eq. (95) becomes important for $r_{3}>c_{s} \sqrt{1-n_{\mathcal{R}}}$. When $n_{\mathcal{R}}=0.96$ this condition translates into $r_{3}>0.2 c_{s}$. If $c_{s}=0.1$ and $r_{3}>0.1$, for example, the non-linear parameter (95) can be larger than the order of 1 . However, for $c_{s}=0.1$, we also expect the dominant contribution to $f_{\mathrm{NL}}$ to come from other shapes (equilateral, orthogonal, etc.)

For the models in which $c_{s}^{2}$ is close to $1, \alpha_{1}$ and $\alpha_{2}$ are of the order of unity. Hence the leading-order non-linear parameter can be estimated as $\left|f_{\mathrm{NL}}^{\text {lead }}\right| \approx r_{3}^{2} \ll 1$ in the regime $r_{3} \ll 1$. By increasing the value of $r_{3}$ from 0 , we can observationally discriminate between the models with $c_{s}^{2} \ll 1$ and $c_{s}^{2} \approx 1$.

\section{B. Equilateral non-Gaussianities}

The equilateral shape is characterized by $k_{1}=k_{2}=k_{3} \equiv k$, in which case the non-linear parameter is $f_{\mathrm{NL}}^{\text {equil }}=$ $(10 / 9)\left(\mathcal{A}_{\mathcal{R}} / k^{3}\right)$. Since $S_{1}=4 k^{3} / 3, S_{2}=17 k^{3} / 6$, and $S_{3}=k^{3} / 27$, the bispectrum (85) gives the leading-order non-linear parameter

$$
\begin{aligned}
f_{\mathrm{NL}}^{\text {equil,lead }}= & \frac{85}{324}\left(1-\frac{1}{c_{s}^{2}}\right)-\frac{10}{81} \frac{\lambda}{\Sigma}+\frac{20}{81 \epsilon_{s}}\left[\left(1+\lambda_{3 X}\right) \delta_{G 3 X}+4\left(3+2 \lambda_{4 X}\right) \delta_{G 4 X X}+\delta_{G 5 X}+\left(5+2 \lambda_{5 X}\right) \delta_{G 5 X X}\right] \\
& +\frac{65}{162 c_{s}^{2} \epsilon_{s}}\left(\delta_{G 3 X}+6 \delta_{G 4 X X}+\delta_{G 5 X}+\delta_{G 5 X X}\right) .
\end{aligned}
$$

If $c_{s}^{2} \ll 1$, then we have $\left|f_{\mathrm{NL}}^{\text {equil,lead }}\right| \gg 1$. 
In the equilateral limit the functions $\mathcal{V}$ and $\mathcal{U}$ are given by $\mathcal{V}=15[1+(1 / 2) \ln (2 / 3)] k^{3}$ and $\mathcal{U}=[6 \ln (3 / 2)-1] k^{3}$, respectively (see Appendix B). Then the functions $\delta \tilde{Q}_{12} \equiv\left(3 \delta Q_{1}+\delta Q_{2}\right) / k^{3}$ and $\delta \tilde{Q}_{3} \equiv \delta Q_{3} / k^{3}$ reduce to

$$
\begin{aligned}
\delta \tilde{Q}_{12}= & \epsilon\left[6-78 \ln (2 / 3)-14 \gamma_{1}\right] / 3-\eta_{s F}\left[7 \gamma_{1}+26 \ln (2 / 3)\right] / 2 \\
& +s[16-39 \ln (2 / 3)] / 3+\tilde{\eta}_{1}\left(7 \gamma_{1}+20\right) / 6, \\
\delta \tilde{Q}_{3}= & -\epsilon\left[10 \gamma_{1}+55+168 \ln (2 / 3)\right] / 27-\eta_{s F}\left[2 \gamma_{1}+7+28 \ln (2 / 3)\right] / 9 \\
& -4 s[10+21 \ln (2 / 3)] / 27+\tilde{\eta}_{3}\left(2 \gamma_{1}-3\right) / 27 .
\end{aligned}
$$

The correction to $f_{\mathrm{NL}}^{\text {equil,lead }}$ coming from Eq. (89) is given by

$$
\begin{aligned}
f_{\mathrm{NL}}^{\text {equil,corre }}=\frac{5}{972 c_{s}^{2} \epsilon_{s}} & {\left[3 \epsilon_{s}\left(24 \delta \mathcal{C}_{1}+51 \delta \mathcal{C}_{2}+4 c_{s}^{2} \delta \mathcal{C}_{3}\right)+8 c_{s}^{2}\left(3 \eta_{s F}-4 s-\eta_{6}\right) \delta \mathcal{C}_{6}+\left(450 \epsilon+327 \eta_{s F}+620 s-497 \eta_{7}\right) \delta \mathcal{C}_{7}\right.} \\
& \left.-153 \epsilon_{s} \delta \mathcal{C}_{8}+72 \epsilon_{s}^{2}\right]-\frac{5}{18}\left(\frac{1}{c_{s}^{2}}-1-\frac{2 \delta \mathcal{C}_{7}}{c_{s}^{2} \epsilon_{s}}\right) \delta \tilde{Q}_{12}+\frac{5}{6}\left(\frac{1}{c_{s}^{2}}-1-\frac{2 \lambda}{\Sigma}+\frac{4 \delta \mathcal{C}_{6}}{\epsilon_{s}}-\frac{4 \delta \mathcal{C}_{7}}{c_{s}^{2} \epsilon_{s}}\right) \delta \tilde{Q}_{3} \cdot(100)
\end{aligned}
$$

For the theories in which $f_{\mathrm{NL}}^{\text {equil,lead }}$ vanishes, the next-order correction $f_{\mathrm{NL}}^{\text {equil,corre }}$ is the dominant contribution. In the case of a canonical scalar field with the Lagrangian $P=X-V(\phi), G_{3}=0, G_{4}=0, G_{5}=0$, for example, it follows that $f_{\mathrm{NL}}^{\text {equil, lead }}=0$ and $f_{\mathrm{NL}}^{\text {equil,corre }}=55 \epsilon_{s} / 36+5 \eta_{s} / 12$.

For the theories with $c_{s}^{2} \neq 1$ the non-linear parameters (97) and (100) reproduce the results known in literature for specific models of inflation. For example, this is the case for k-inflation [18, 56], k-inflation with the Galileon terms 62, 63], potential-driven Galileon inflation [73], and inflation with a field derivative coupling to the Einstein tensor [53]. Generally we require that $c_{s}^{2} \ll 1$ to realize the large equilateral non-linear parameter.

\section{Enfolded non-Gaussianities}

The enfolded shape is characterized by $k_{2}+k_{3}=k_{1}$. Taking the momenta $k_{1}=k$ and $k_{2} \rightarrow k_{3}=k / 2$, the non-linear parameter ${ }^{4}$ is $f_{\mathrm{NL}}^{\text {enfold }}=8 \mathcal{A}_{\mathcal{R}} /\left(3 k^{3}\right)$. Since $S_{1}=23 k^{3} / 64, S_{2}=63 k^{3} / 64$, and $S_{3}=k^{3} / 128$ in this case, the leading-order non-linear parameter is given by

$$
f_{\mathrm{NL}}^{\text {enfold,lead }}=\frac{1}{32}\left(1-\frac{1}{c_{s}^{2}}\right)-\frac{1}{16} \frac{\lambda}{\Sigma}+\frac{1}{8 \epsilon_{s}}\left[\left(1+\lambda_{3 X}\right) \delta_{G 3 X}+4\left(3+2 \lambda_{4 X}\right) \delta_{G 4 X X}+\delta_{G 5 X}+\left(5+2 \lambda_{5 X}\right) \delta_{G 5 X X}\right],
$$

where, unlike the equilateral case, the $\delta \mathcal{C}_{7}$-dependent term in Eq. (85) disappears.

In the enfolded limit one has $\mathcal{V}=[315 / 64-\ln (2) / 2] k^{3}$ and $\mathcal{U}=[5 / 24+\ln (2) / 4] k^{3}$ (see Appendix B). The functions $\delta \tilde{Q}_{12}$ and $\delta \tilde{Q}_{3}$ are

$$
\begin{aligned}
\delta \tilde{Q}_{12}= & \epsilon\left[45-24 \gamma_{1}+108 \ln (2)\right] / 64-\eta_{s F}\left[87+36 \gamma_{1}-108 \ln (2)\right] / 128 \\
& +s[333+108 \ln (2)] / 128+\tilde{\eta}_{1}\left(39+3 \gamma_{1}\right) / 32, \\
\delta \tilde{Q}_{3}= & -\epsilon\left[15 \gamma_{1}+17-54 \ln (2)\right] / 192-\eta_{s F}\left[36 \gamma_{1}-5-108 \ln (2)\right] / 768 \\
& -s[109-108 \ln (2)] / 768+\tilde{\eta}_{3}\left(2 \gamma_{1}-3\right) / 128 .
\end{aligned}
$$

The correction to $f_{\mathrm{NL}}^{\text {enfold,lead }}$ is

$$
\begin{aligned}
f_{\mathrm{NL}}^{\text {enfold,corre }}= & \frac{1}{96 c_{s}^{2} \epsilon_{s}}\left[\epsilon_{s}\left(23 \delta \mathcal{C}_{1}+63 \delta \mathcal{C}_{2}+3 c_{s}^{2} \delta \mathcal{C}_{3}\right)-2 c_{s}^{2}\left(\eta_{6}+4 s-3 \eta_{s F}\right) \delta \mathcal{C}_{6}+\left(172 \epsilon+254 s+92 \eta_{s F}-174 \eta_{7}\right) \delta \mathcal{C}_{7}\right. \\
& \left.+2 \epsilon_{s}^{2}\right]-\frac{2}{3}\left(\frac{1}{c_{s}^{2}}-1-\frac{2 \delta \mathcal{C}_{7}}{c_{s}^{2} \epsilon_{s}}\right) \delta \tilde{Q}_{12}+2\left(\frac{1}{c_{s}^{2}}-1-\frac{2 \lambda}{\Sigma}+\frac{4 \delta \mathcal{C}_{6}}{\epsilon_{s}}-\frac{4 \delta \mathcal{C}_{7}}{c_{s}^{2} \epsilon_{s}}\right) \delta \tilde{Q}_{3}
\end{aligned}
$$

For a canonical scalar field we have that $f_{\mathrm{NL}}^{\text {enfold,lead }}=0$ and $f_{\mathrm{NL}}^{\text {enfold,corre }}=7 \epsilon_{s} / 8+5 \eta_{s} / 12$.

\footnotetext{
${ }^{4}$ At the point $k_{1}=k$ and $k_{2} \rightarrow k_{3}=k / 2$, the equilateral shape gives no contribution. However, the contribution from the orthogonal shape will be of the same order of the enfolded one, since, by definition, in this case we have $f_{\mathrm{NL}}^{\text {ortho }} \rightarrow-2 f_{\mathrm{NL}}^{\text {enfold }}$.
} 


\section{SHAPES OF NON-GAUSSIANITIES}

The leading-order bispectrum (47) can be written in terms of the sum of each component, as $\mathcal{A}_{\mathcal{R}}=\sum_{i=1}^{8} \mathcal{A}_{\mathcal{R}}^{(i)}$. Then the function $\mathcal{F}_{\mathcal{R}}\left(k_{1}, k_{2}, k_{3}\right)$ in Eq. (46) is decomposed into eight components

$$
\mathcal{F}_{\mathcal{R}}^{(i)}=(2 \pi)^{4} \frac{\mathcal{A}_{\mathcal{R}}^{(i)}}{\prod_{i=1}^{3} k_{i}^{3}}=\frac{(2 \pi)^{4}}{\left(k_{1} k_{2} k_{3}\right)^{3}} \mathcal{B}_{i} S_{i},
$$

where $\mathcal{B}_{i}$ 's are the coefficients appearing in front of each shape function $S_{i}$ in Eq. (47), say, $\mathcal{B}_{1}=c_{s}^{2} \mathcal{C}_{1} /\left(4 \epsilon_{s} F\right)$.

In order to estimate the correlation between two different shapes, we define the following quantity [42]

$$
C\left(\mathcal{F}_{\mathcal{R}}^{(i)}, \mathcal{F}_{\mathcal{R}}^{(j)}\right)=\frac{\mathcal{I}\left(\mathcal{F}_{\mathcal{R}}^{(i)}, \mathcal{F}_{\mathcal{R}}^{(j)}\right)}{\sqrt{\mathcal{I}\left(\mathcal{F}_{\mathcal{R}}^{(i)}, \mathcal{F}_{\mathcal{R}}^{(i)}\right) \mathcal{I}\left(\mathcal{F}_{\mathcal{R}}^{(j)}, \mathcal{F}_{\mathcal{R}}^{(j)}\right)}},
$$

where

$$
\mathcal{I}\left(\mathcal{F}_{\mathcal{R}}^{(i)}, \mathcal{F}_{\mathcal{R}}^{(j)}\right)=\int d \mathcal{V}_{k} \mathcal{F}_{\mathcal{R}}^{(i)}\left(k_{1}, k_{2}, k_{3}\right) \mathcal{F}_{\mathcal{R}}^{(j)}\left(k_{1}, k_{2}, k_{3}\right) \frac{\left(k_{1} k_{2} k_{3}\right)^{4}}{\left(k_{1}+k_{2}+k_{3}\right)^{3}}
$$

The integration should be done in the region $0 \leq k_{1}<\infty, 0<k_{2} / k_{1}<1$, and $1-k_{2} / k_{1} \leq k_{3} / k_{1} \leq 1$. Note that the above integral can be expressed in terms of $r_{2}=k_{2} / k_{1}$ and $r_{3}=k_{3} / k_{1}$ with the integral of $k_{1}$ factorized out. For $\left|C\left(\mathcal{F}_{\mathcal{R}}^{(i)}, \mathcal{F}_{\mathcal{R}}^{(j)}\right)\right|$ close to 1 the correlation is large, whereas for $\left|C\left(\mathcal{F}_{\mathcal{R}}^{(i)}, \mathcal{F}_{\mathcal{R}}^{(j)}\right)\right|$ close to 0 the two shapes are almost orthogonal with a small correlation.

The CMB data analysis of non-Gaussianities has been carried out by using the factorizable shape functions which are written as the sums of monomials of $k_{1}, k_{2}$, and $k_{3}$. There are a number of templates $\mathcal{F}_{\mathcal{R}}$ which resemble model predictions of the bispectrum. The templates corresponding to local and equilateral non-Gaussianities are given, respectively, by [37, 41]

$$
\mathcal{F}_{\mathcal{R}}^{\text {local }}\left(k_{1}, k_{2}, k_{3}\right)=(2 \pi)^{4}\left(\frac{3}{10} f_{\mathrm{NL}}^{\text {local }}\right)\left(\frac{1}{k_{1}^{3} k_{2}^{3}}+\frac{1}{k_{2}^{3} k_{3}^{3}}+\frac{1}{k_{3}^{3} k_{1}^{3}}\right)
$$

and

$$
\mathcal{F}_{\mathcal{R}}^{\text {equil }}\left(k_{1}, k_{2}, k_{3}\right)=(2 \pi)^{4}\left(\frac{9}{10} f_{\mathrm{NL}}^{\text {equil }}\right)\left[-\frac{1}{k_{1}^{3} k_{2}^{3}}-\frac{1}{k_{2}^{3} k_{3}^{3}}-\frac{1}{k_{3}^{3} k_{1}^{3}}-\frac{2}{k_{1}^{2} k_{2}^{2} k_{3}^{2}}+\left(\frac{1}{k_{1} k_{2}^{2} k_{3}^{3}}+5 \text { perm. }\right)\right] .
$$

Since the local non-Gaussianities are small in the Horndeski's theories, we do not consider the correlation with the local template.

The orthogonal template, which has a small correlation with the equilateral one, is given by [43]

$$
\mathcal{F}_{\mathcal{R}}^{\text {ortho }}\left(k_{1}, k_{2}, k_{3}\right)=(2 \pi)^{4}\left(\frac{9}{10} f_{\mathrm{NL}}^{\text {ortho }}\right)\left[-\frac{3}{k_{1}^{3} k_{2}^{3}}-\frac{3}{k_{2}^{3} k_{3}^{3}}-\frac{3}{k_{3}^{3} k_{1}^{3}}-\frac{8}{k_{1}^{2} k_{2}^{2} k_{3}^{2}}+\left(\frac{3}{k_{1} k_{2}^{2} k_{3}^{3}}+5 \text { perm. }\right)\right] .
$$

The enfolded template, which is a linear combination of the orthogonal and equilateral templates, is defined by [44]

$$
\mathcal{F}_{\mathcal{R}}^{\text {enfold }}\left(k_{1}, k_{2}, k_{3}\right)=(2 \pi)^{4}\left(\frac{9}{10} f_{\mathrm{NL}}^{\mathrm{enf}}\right)\left[\frac{1}{k_{1}^{3} k_{2}^{3}}+\frac{1}{k_{2}^{3} k_{3}^{3}}+\frac{1}{k_{3}^{3} k_{1}^{3}}+\frac{3}{k_{1}^{2} k_{2}^{2} k_{3}^{2}}-\left(\frac{1}{k_{1} k_{2}^{2} k_{3}^{3}}+5 \text { perm. }\right)\right] .
$$

In Table \we show the correlation between $\mathcal{F}_{\mathcal{R}}^{(i)}(i=3,4,5,7,8)$ and the three templates given above. Since the correlations between $\mathcal{F}_{\mathcal{R}}^{(i)}(i=4,5,7,8)$ and $\mathcal{F}_{\mathcal{R}}^{\text {equil }}$ are close to $1, \mathcal{F}_{\mathcal{R}}^{(i)}(i=4,5,7,8)$ are well approximated by the equilateral shape. In particular, both $C\left(\mathcal{F}_{\mathcal{R}}^{(7)}, \mathcal{F}_{\mathcal{R}}^{\text {ortho }}\right)$ and $C\left(\mathcal{F}_{\mathcal{R}}^{(8)}, \mathcal{F}_{\mathcal{R}}^{\text {ortho }}\right)$ are close to 0 with the same level of correlation between $\mathcal{F}_{\mathcal{R}}^{\text {equil }}$ and $\mathcal{F}_{\mathcal{R}}^{\text {ortho }}$. Hence the shape functions $S_{7}$ and $S_{8}$ highly mimic the equilateral template. They also vanish in the local limit $\left(k_{3} \rightarrow 0\right)$ and in the enfolded limit $\left(k_{2}+k_{3} \rightarrow k_{1}\right)$. Note that both $\left|C\left(\mathcal{F}_{\mathcal{R}}^{(7)}, \mathcal{F}_{\mathcal{R}}^{\text {enfold }}\right)\right|$ and $\left|C\left(\mathcal{F}_{\mathcal{R}}^{(8)}, \mathcal{F}_{\mathcal{R}}^{\text {enfold }}\right)\right|$ are close to 0.5 (which is similar to the value $\left|C\left(\mathcal{F}_{\mathcal{R}}^{\text {equil }}, \mathcal{F}_{\mathcal{R}}^{\text {enfold }}\right)\right|=0.511911$ ). Since there is the relation $\mathcal{F}_{\mathcal{R}}^{\text {enfold }}=\left(\mathcal{F}_{\mathcal{R}}^{\text {equil }}-\mathcal{F}_{\mathcal{R}}^{\text {ortho }}\right) / 2$, we have $\left|C\left(\mathcal{F}_{\mathcal{R}}^{(i)}, \mathcal{F}_{\mathcal{R}}^{\text {enfold }}\right)\right| \simeq 0.5$ for the shape function $\mathcal{F}_{\mathcal{R}}^{(i)}$ very close to the equilateral one. 


\begin{tabular}{|c|c|c|c|c|c|c|c|c|c|c|}
\hline & $\mathcal{F}_{\mathcal{R}}^{\text {equil }}$ & $\mathcal{F}_{\mathcal{R}}^{\text {ortho }}$ & $\mathcal{F}_{\mathcal{R}}^{\text {enfold }}$ & $\mathcal{F}_{\mathcal{R}}^{(3)}$ & $\mathcal{F}_{\mathcal{R}}^{(4)}$ & $\mathcal{F}_{\mathcal{R}}^{(5)}$ & $\mathcal{F}_{\mathcal{R}}^{(7 \text { equil })}$ & $\mathcal{F}_{\mathcal{R}}^{(8)}$ & $\mathcal{F}_{\mathcal{R}}^{\text {(7ortho) }}$ & $3 \mathcal{F}_{\mathcal{R}}^{(1)}-\mathcal{F}_{\mathcal{R}}^{(2)}$ \\
\hline $\mathcal{F}_{\mathcal{R}}^{\text {equil }}$ & 1 & 0.0254062 & 0.511911 & 0.936177 & -0.998757 & -0.994234 & 0.999892 & -0.999994 & -0.00693357 & 0.986954 \\
\hline $\mathcal{F}_{\mathcal{R}}^{\text {ortho }}$ & & 1 & -0.845755 & -0.290742 & 0.0177139 & -0.117961 & 0.0353534 & -0.0277283 & 0.904843 & -0.116557 \\
\hline $\mathcal{F}_{\mathcal{R}}^{\text {enfold }}$ & & & 1 & \begin{tabular}{|l|}
0.749518 \\
\end{tabular} & \begin{tabular}{|l}
-0.548302 \\
\end{tabular} & -0.4293 & 0.503306 & -0.509913 & -0.781246 & 0.626939 \\
\hline $\mathcal{F}_{\mathcal{R}}^{(3)}$ & & & & 1 & -0.952469 & -0.893224 & 0.933797 & -0.935615 & -0.357802 & 0.980504 \\
\hline $\mathcal{F}_{\mathcal{R}}^{(4)}$ & & & & & 1 & 0.987653 & -0.998384 & 0.998686 & 0.056402 & -0.993745 \\
\hline $\mathcal{F}_{\mathcal{R}}^{(5)}$ & & & & & & 1 & -0.994696 & 0.99437 & -0.0995524 & -0.964012 \\
\hline $\mathcal{F}_{\mathcal{R}}^{\text {(7equil) }}$ & & & & & & & 1 & -0.999936 & 0 & 0.9859 \\
\hline $\mathcal{F}_{\mathcal{R}}^{(8)}$ & & & & & & & & 1 & 0.00524865 & -0.986715 \\
\hline $\mathcal{F}_{\mathcal{R}}^{\text {(7ortho) }}$ & & & & & & & & & 1 & -0.167335 \\
\hline
\end{tabular}

Table I: The correlation (106) between two different shape functions. $\mathcal{F}_{\mathcal{R}}^{(7 \text { equil) }}$ is the normalized shape of $\mathcal{F}_{\mathcal{R}}^{(7)}$, whereas $\mathcal{F}_{\mathcal{R}}^{(7 \text { ortho) }}$ is the shape function orthogonal to $\mathcal{F}_{\mathcal{R}}^{(7 \text { equil) }}$ (or, equivalently, to $\mathcal{F}_{\mathcal{R}}^{(7)}$ ).

We recall that the functions $3 S_{1}-S_{2}$ and $S_{3}$ are related with $S_{7}$ [see Eq. (49)]. We can use this property in order to rewrite the leading-order part of the bispectrum in a convenient basis. We introduce the following shape

$$
S_{7}^{\text {equil }}=-\frac{12}{13} S_{7}
$$

whose minus sign has been chosen so that $\mathcal{F}_{\mathcal{R}}^{(7 \text { equil) }}$ has a positive high correlation with the equilateral profile. Furthermore, we can analytically show that the following shape is exactly orthogonal to $S_{7}^{\text {equil. }}$

$$
S_{7}^{\text {ortho }}=\frac{12}{14-13 \beta}\left(\beta S_{7}+3 S_{1}-S_{2}\right),
$$

where

$$
\beta=\frac{16}{3} \frac{248041-25200 \pi^{2}}{1986713-201600 \pi^{2}}=1.1967996 \ldots
$$

The normalizations of $S_{7}^{\text {equil }}$ and $S_{7}^{\text {ortho }}$ have been done such that, at the equilateral configuration $\left(k_{1}=k_{2}=k_{3}=k\right)$, we have $S_{7}^{\text {equil }}=k^{3}=S_{7}^{\text {ortho }}$. This normalization follows from the standard definition of the previous templates introduced in the literature.

We note here that the leading-order bispectrum includes the term $3 S_{1}-S_{2}$, so that we also consider the correlation between the combination $3 \mathcal{F}_{\mathcal{R}}^{(1)}-\mathcal{F}_{\mathcal{R}}^{(2)}$ and other shapes in Table I. The shape $3 \mathcal{F}_{\mathcal{R}}^{(1)}-\mathcal{F}_{\mathcal{R}}^{(2)}$ has a high correlation with $\mathcal{F}_{\mathcal{R}}^{\text {equil }}$. Compared to $\mathcal{F}_{\mathcal{R}}^{(7)}$ and $\mathcal{F}_{\mathcal{R}}^{(8)}$, however, it is not very close to the equilateral shape. Moreover $\mathcal{F}_{\mathcal{R}}^{(3)}$ has some correlation with the orthogonal shape, i.e., $C\left(\mathcal{F}_{\mathcal{R}}^{(3)}, \mathcal{F}_{\mathcal{R}}^{\text {ortho }}\right)=-0.290742$.

As we see in Table I, the correlation between $\mathcal{F}_{\mathcal{R}}^{\text {(7ortho) }}$ and $\mathcal{F}_{\mathcal{R}}^{\text {equil }}$ is very small. In terms of $S_{7}^{\text {equil }}$ and $S_{7}^{\text {ortho }}$, the functions $3 S_{1}-S_{2}$ and $S_{3}$ are expressed as

$$
3 S_{1}-S_{2}=\frac{13}{12} \beta S_{7}^{\text {equil }}+\frac{14-13 \beta}{12} S_{7}^{\text {ortho }}, \quad S_{3}=\frac{13}{432}(3 \beta-2) S_{7}^{\text {equil }}+\frac{14-13 \beta}{144} S_{7}^{\text {ortho }} .
$$

Using these relations, the leading-order bispectrum (85) can be written in terms of the equilateral basis $S_{7}^{\text {equil }}$ and the orthogonal basis $S_{7}^{\text {ortho }}$, as

$$
\mathcal{A}_{\mathcal{R}}^{\text {lead }}=c_{1} S_{7}^{\text {equil }}+c_{2} S_{7}^{\text {ortho }}
$$

where

$$
\begin{aligned}
c_{1} & =\frac{13}{12}\left[\frac{1}{24}\left(1-\frac{1}{c_{s}^{2}}\right)(2+3 \beta)+\frac{\lambda}{12 \Sigma}(2-3 \beta)-\frac{\delta \mathcal{C}_{6}}{6 \epsilon_{s}}(2-3 \beta)+\frac{\delta \mathcal{C}_{7}}{3 \epsilon_{s} c_{s}^{2}}\right] \\
c_{2} & =\frac{14-13 \beta}{12}\left[\frac{1}{8}\left(1-\frac{1}{c_{s}^{2}}\right)-\frac{\lambda}{4 \Sigma}+\frac{\delta \mathcal{C}_{6}}{2 \epsilon_{s}}\right] .
\end{aligned}
$$

The coefficients $c_{1}$ and $c_{2}$ characterize the magnitudes of the three-point correlation function coming from equilateral and orthogonal contributions, respectively. Finally, we also introduce the enfolded shape function

$$
S_{7}^{\text {enfold }}=\left(S_{7}^{\text {equil }}-S_{7}^{\text {ortho }}\right) / 2
$$




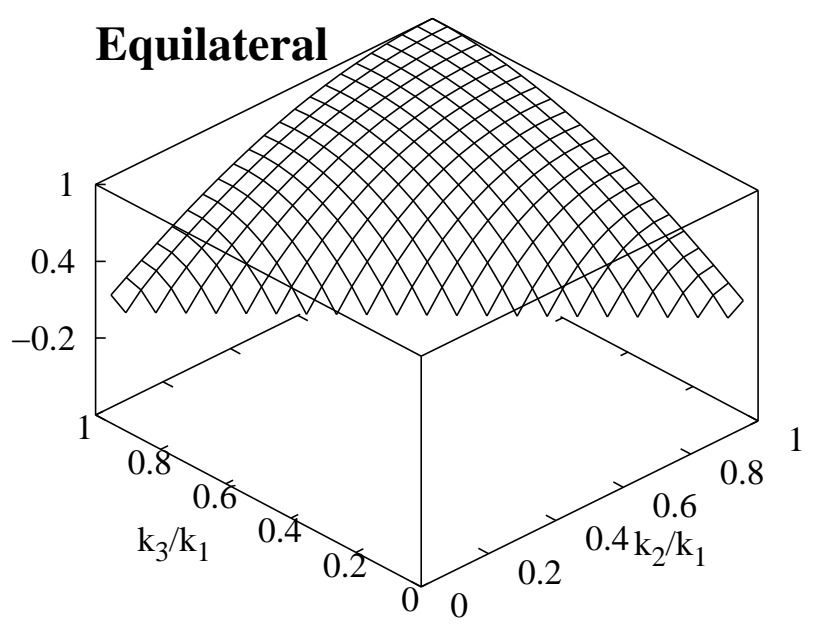

\section{Orthogonal}

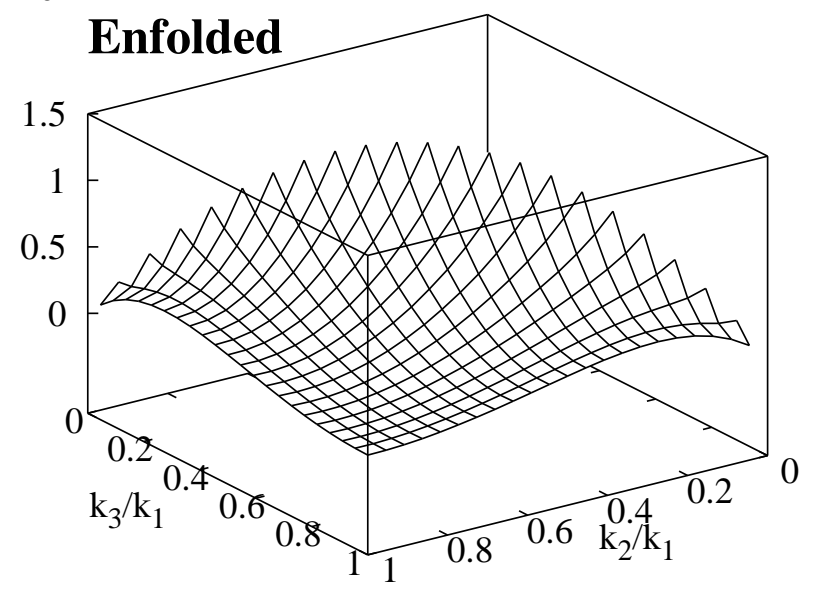

Figure 1: The shape functions $\mathcal{F}_{\mathcal{R}}^{\text {(7equil) }}\left(1, k_{2} / k_{1}, k_{3} / k_{1}\right)\left(k_{2} / k_{1}\right)^{2}\left(k_{3} / k_{1}\right)^{2}$ (top left), $\mathcal{F}_{\mathcal{R}}^{\text {(7ortho) }}\left(1, k_{2} / k_{1}, k_{3} / k_{1}\right)\left(k_{2} / k_{1}\right)^{2}\left(k_{3} / k_{1}\right)^{2}$ (top right), and $\mathcal{F}_{\mathcal{R}}^{\text {(7enfold) }}\left(1, k_{2} / k_{1}, k_{3} / k_{1}\right)\left(k_{2} / k_{1}\right)^{2}\left(k_{3} / k_{1}\right)^{2}$ (bottom).

which has a maximum at $k_{1} \rightarrow 2 k_{2}, k_{2} \rightarrow k_{3}$. Note that $S_{7}^{\text {enfold }}$ vanishes at the equilateral configuration.

In Fig. 1 we plot the three shape functions $\mathcal{F}_{\mathcal{R}}^{\text {(7equil) }}, \mathcal{F}_{\mathcal{R}}^{\text {(7ortho) }}$ and $\mathcal{F}_{\mathcal{R}}^{\text {(7enfold) }}$ multiplied by the functions $\left(k_{2} / k_{1}\right)^{2}\left(k_{3} / k_{1}\right)^{2}$. The correlations of $\mathcal{F}_{\mathcal{R}}^{\text {lead }}=(2 \pi)^{4} \mathcal{A}_{\mathcal{R}}^{\text {lead }} / \prod_{i=1}^{3} k_{i}^{3}$ with the shape functions $\mathcal{F}_{\mathcal{R}}^{\text {equil }}$, $\mathcal{F}_{\mathcal{R}}^{\text {ortho }}$, and $\mathcal{F}_{\mathcal{R}}^{\text {enfold }}$ can be evaluated as

$$
\begin{gathered}
C^{\text {equil }} \equiv C\left(\mathcal{F}_{\mathcal{R}}^{\text {lead }}, \mathcal{F}_{\mathcal{R}}^{\text {equil }}\right)=\frac{8.25104\left(1.44717 \times 10^{-2} c_{1}-1.70045 \times 10^{-4} c_{2}\right)}{\sqrt{1.42610 \times 10^{-2} c_{1}^{2}+4.09480 \times 10^{-2} c_{2}^{2}}}, \\
C^{\text {ortho }} \equiv C\left(\mathcal{F}_{\mathcal{R}}^{\text {lead }}, \mathcal{F}_{\mathcal{R}}^{\text {ortho }}\right)=\frac{5.12494\left(8.23792 \times 10^{-4} c_{1}+3.57273 \times 10^{-2} c_{2}\right)}{\sqrt{1.42610 \times 10^{-2} c_{1}^{2}+4.09480 \times 10^{-2} c_{2}^{2}}}, \\
C^{\text {enfold }} \equiv C\left(\mathcal{F}_{\mathcal{R}}^{\text {lead }}, \mathcal{F}_{\mathcal{R}}^{\text {enfold }}\right)=\frac{8.80788\left(6.82395 \times 10^{-3} c_{1}-1.79487 \times 10^{-2} c_{2}\right)}{\sqrt{1.42610 \times 10^{-2} c_{1}^{2}+4.09480 \times 10^{-2} c_{2}^{2}}},
\end{gathered}
$$

which depend on the coefficients $c_{1}$ and $c_{2}$. In particular, we find $C\left(\mathcal{F}_{\mathcal{R}}^{(7 \text { enfold })}, \mathcal{F}_{\mathcal{R}}^{\text {enfold }}\right)=0.928621$.

\section{SHAPES OF NON-GAUSSIANITIES IN CONCRETE MODELS}

Let us study the non-Gaussianities of concrete models of inflation in which the bispectrum (116) can be large due to the small scalar propagation speed $c_{s}$. As we will see below, there are some models where the orthogonal shape provides an important contribution to the bispectrum. 


\section{A. Power-law k-inflation}

We first consider k-inflation characterized by

$$
P(\phi, X)=K(\phi)\left(-X+X^{2}\right), \quad G_{3}=0, \quad G_{4}=0, \quad G_{5}=0,
$$

where $K(\phi)$ is a function in terms of $\phi$. From the background equations (9) and (10) it follows that

$$
K(\phi)=\frac{3 M_{\mathrm{pl}}^{2} H^{2}}{X(3 X-1)}, \quad \frac{\dot{H}}{H^{2}}=-\frac{3(2 X-1)}{3 X-1} .
$$

As an example, we study power-law inflation characterized by $a \propto t^{1 / \gamma}$ and $H=1 /(\gamma t)$, where $\gamma(\ll 1)$ is constant. Substituting the Hubble parameter into the second of Eq. (124), we obtain $X=(3-\gamma) /[3(2-\gamma)]$ and $\phi=\phi_{0}+$ $\sqrt{(3-\gamma) /[3(2-\gamma)]} t\left(\phi_{0}\right.$ is the initial value of the field). From the first of Eq. (124) we find that power-law inflation is realized for the choice [54]

$$
K(\phi)=\frac{6(2-\gamma) M_{\mathrm{pl}}^{2}}{\gamma^{2}\left(\phi-\phi_{0}\right)^{2}}
$$

In this case $c_{s}^{2}$ and $\lambda / \Sigma$ in Eq. (116) are given by

$$
c_{s}^{2}=\frac{\gamma}{3(4-\gamma)}, \quad \frac{\lambda}{\Sigma}=\frac{1}{2}\left(1-c_{s}^{2}\right) .
$$

For $\gamma \ll 1$ we have that $1 / c_{s}^{2} \simeq 12 / \gamma \gg 1$.

In this model (dubbed Model A) the leading-order bispectrum (116) reduces to

$$
\mathcal{A}_{\mathcal{R}}^{\text {lead }}=\frac{13}{12}\left[-\frac{2+3 \beta}{24 c_{s}^{2}}+\frac{1}{6}-\frac{1}{24}(2-3 \beta) c_{s}^{2}\right] S_{7}^{\text {equil }}-\frac{14-13 \beta}{96}\left(\frac{1}{c_{s}^{2}}-c_{s}^{2}\right) S_{7}^{\text {ortho }} .
$$

In the limit $c_{s}^{2} \ll 1$ it follows that $\mathcal{A}_{\mathcal{R}}^{\text {lead }} \simeq\left(-0.252 / c_{s}^{2}\right) S_{7}^{\text {equil }}+\left(0.016 / c_{s}^{2}\right) S_{7}^{\text {ortho }}$. Since in this limit the non-linear

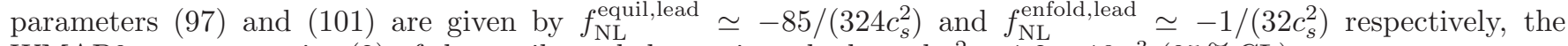
WMAP9 year constraint (2) of the equilateral shape gives the bound $c_{s}^{2}>1.2 \times 10^{-3}$ (95\% CL).

The scalar spectral index (29) and the tensor-to-scalar ratio (31) can be expressed in terms of $c_{s}$, as

$$
n_{\mathcal{R}}=1-\frac{24 c_{s}^{2}}{1+3 c_{s}^{2}}, \quad r=\frac{192 c_{s}^{3}}{1+3 c_{s}^{2}}
$$

For the $\Lambda \mathrm{CDM}$ model without the running scalar spectral index, the bounds on $n_{s}$ and $r$ from the WMAP9 data alone are $n_{\mathcal{R}}=0.992 \pm 0.019(68 \%$ CL) and $r<0.38$ (95\% CL), respectively. If we combine the WMAP9 data with the measurements of high- $l$ CMB anisotropies, baryon acoustic oscillations, and the Hubble constant, the constraints are $0.9636 \pm 0.0084(68 \% \mathrm{CL})$ and $r<0.13(95 \% \mathrm{CL})$. If we employ the bound $0.95<n_{\mathcal{R}}<1$ then the scalar propagation is constrained to be $c_{s}^{2}<2.1 \times 10^{-3}$. Since in this case $r<0.018$ from Eq. (128), the observational constraint on $r$ is satisfied. Combining the bound of $c_{s}^{2}$ with that of the scalar non-Gaussianity, it follows that $1.2 \times 10^{-3}<c_{s}^{2}<2.1 \times 10^{-3}$.

In Fig. 2 2 we plot the shape function $\mathcal{F}_{\mathcal{R}}^{\text {lead }}\left(1, k_{2} / k_{1}, k_{3} / k_{1}\right)\left(k_{2} / k_{1}\right)^{2}\left(k_{3} / k_{1}\right)^{2}$ for $c_{s}^{2}=2.0 \times 10^{-3}$ (labelled as "A" in the figure), where $\mathcal{F}_{\mathcal{R}}^{\text {lead }}=(2 \pi)^{4} \mathcal{A}_{\mathcal{R}}^{\text {lead }} / \prod_{i=1}^{3} k_{i}^{3}$. When $c_{s}^{2}=2.0 \times 10^{-3}$, the correlations (120)-(122) are $C^{\text {equil }}=-0.99474$, $C^{\text {ortho }}=0.06305$, and $C^{\text {enfold }}=-0.58511$, respectively. Hence the shape of non-Gaussianities is close to the equilateral one illustrated in Fig. 1] whose property is is independent of the choice of $c_{s}^{2}$.

\section{B. k-inflation with the term $G_{3}(X)$}

We study k-inflation in the presence of the covariant Galileon term $G_{3}(X)$ characterized by [63, 71]

$$
P(X)=-X+\frac{X^{2}}{2 M^{4}}, \quad G_{3}(X)=\frac{\mu X}{M^{4}},
$$


where $M$ and $\mu$ are constants having a dimension of mass. In this model (dubbed Model B) the de Sitter solution is present when $\epsilon=\delta_{P X}+3 \delta_{G 3 X}=0$. Using Eq. (9) as well, we obtain

$$
H^{2}=\frac{M^{4}}{18 \mu^{2}} \frac{(1-x)^{2}}{x}, \quad \frac{\mu}{M_{\mathrm{pl}}}=\frac{1-x}{x \sqrt{3(2-x)}},
$$

where $x=X / M^{4}$. As long as inflation is realized in the regime $\mu / M_{\mathrm{pl}} \ll 1, x$ is close to 1 . In what follows we replace $x$ for 1 except for the terms including $1-x$.

Along the de Sitter solution we have that $\lambda / \Sigma=6 c_{s}^{2} / \epsilon_{s}, \delta \mathcal{C}_{6}=\delta \mathcal{C}_{7}=\delta_{G 3 X}=2(1-x)$. Since $\epsilon_{s}=2(1-x)$ and $c_{s}^{2}=(1-x) / 6$, the bispectrum (116) reads

$$
\mathcal{A}_{\mathcal{R}}^{\text {lead }}=\frac{13}{12}\left[\frac{1}{4 c_{s}^{2}}\left(1-\frac{\beta}{2}\right)-\frac{1}{6}(1-3 \beta)\right] S_{7}^{\text {equil }}-\frac{14-13 \beta}{12}\left(\frac{1}{8 c_{s}^{2}}-\frac{1}{2}\right) S_{7}^{\text {ortho }} .
$$

If $c_{s}^{2} \ll 1$, we obtain $\mathcal{A}_{\mathcal{R}}^{\text {lead }} \simeq\left(0.109 / c_{s}^{2}\right) S_{7}^{\text {equil }}+\left(0.016 / c_{s}^{2}\right) S_{7}^{\text {ortho }}$ together with the non-linear parameters $f_{\mathrm{NL}}^{\text {equil,lead }} \simeq$ $5 /\left(36 c_{s}^{2}\right)$ and $f_{\mathrm{NL}}^{\text {enfold,lead }} \simeq-1 /\left(32 c_{s}^{2}\right)$. Notice that the sign of $f_{\mathrm{NL}}^{\text {equil,lead }}$ is opposite compared to the k-inflation model A. From the WMAP 9 year bound (2) it follows that $c_{s}^{2}>4.3 \times 10^{-4}(95 \% \mathrm{CL})$. In Model B the scalar spectral index is $n_{\mathcal{R}}=1$, whereas $r$ is related to $c_{s}^{2}$ via

$$
c_{s}^{2}=\frac{3^{1 / 3}}{48} r^{2 / 3} .
$$

The WMAP9 bound $r<0.38$ gives the constraint $c_{s}^{2}<1.6 \times 10^{-2}$. Using the severer bound $r<0.13$, it follows that $c_{s}^{2}<7.7 \times 10^{-3}$. In both cases there are viable parameter spaces compatible with the constraint from the scalar nonGaussainity. When $c_{s}^{2}=2.0 \times 10^{-3}$, for example, we have $C^{\text {equil }}=0.96865, C^{\text {ortho }}=0.25272$, and $C^{\text {enfold }}=0.29984$. As we see in Fig. 2. the shape of non-Gaussianities for $c_{s}^{2}=2.0 \times 10^{-3}$ is approximately close to the equilateral one.

For potential-driven inflation $(P=X-V(\phi))$ with the term $G_{3}(X)=\mu X / M^{4}$ [72], the non-Gaussianities are small because $c_{s}^{2}$ is not much smaller than 1 [73]. In such models, if the Galileon self-interaction dominates over the standard kinetic term even after inflation, there is an instability associated with the appearance of the negative $c_{s}^{2}$ after the field velocity $\dot{\phi}$ changes its sign $[74]^{5}$. In the model B discussed above, reheating needs to occur gravitationally [71], so the situation should be different from that in Galileon inflation driven by a potential with a minimum.

\section{C. k-inflation with the term $G_{4}(X)$}

The next model (dubbed Model C) is k-inflation with the covariant Galileon term $G_{4}(X)$ [62], i.e.,

$$
P(X)=-X+\frac{X^{2}}{2 M^{4}}, \quad G_{4}(X)=\frac{\mu X^{2}}{M^{7}} .
$$

Similar to the case (129), there is a de Sitter solution satisfying the conditions

$$
H^{2}=\frac{M^{3}}{36 \mu} \frac{1-x}{x}, \quad \frac{\mu M}{M_{\mathrm{pl}}^{2}}=\frac{1-x}{6 x^{2}(3-2 x)} .
$$

Inflation occurs in the regime where $x=X / M^{4}$ is close to 1 . Employing the similar approximation to that used previously, we have $\delta \mathcal{C}_{6}=2 \delta \mathcal{C}_{7}=12 \delta_{G 4 X X}, \epsilon_{s}=8 \delta_{G 4 X X}, \delta_{G 4 X X}=(1-x) / 3, c_{s}^{2}=2(1-x) / 9$, and $\lambda / \Sigma=1 / 2$. Hence the bispectrum (116) reduces to

$$
\mathcal{A}_{\mathcal{R}}^{\text {lead }}=\frac{13}{12}\left[\frac{1}{24 c_{s}^{2}}(4-3 \beta)-\frac{1}{12}(4-9 \beta)\right] S_{7}^{\text {equil }}-\frac{14-13 \beta}{12}\left(\frac{1}{8 c_{s}^{2}}-\frac{3}{4}\right) S_{7}^{\text {ortho }} .
$$

In the limit $c_{s}^{2} \ll 1$, we have $\mathcal{A}_{\mathcal{R}}^{\text {lead }} \simeq\left(0.018 / c_{s}^{2}\right) S_{7}^{\text {equil }}+\left(0.016 / c_{s}^{2}\right) S_{7}^{\text {ortho }}$. The equilateral and orthogonal non-linear parameters in this limit are given by $f_{\mathrm{NL}}^{\text {equil,lead }} \simeq 25 /\left(648 c_{s}^{2}\right)$ and $f_{\mathrm{NL}}^{\text {enfold,lead }} \simeq-1 /\left(32 c_{s}^{2}\right)$, respectively. The tighter

\footnotetext{
${ }^{5}$ In the framework of the effective field theory the Lagrangian is valid at the energy scale of inflation, but some higher dimensional operators can appear during the reheating stage. There may be a possibility that the Laplacian instability can be avoided by such operators.
} 

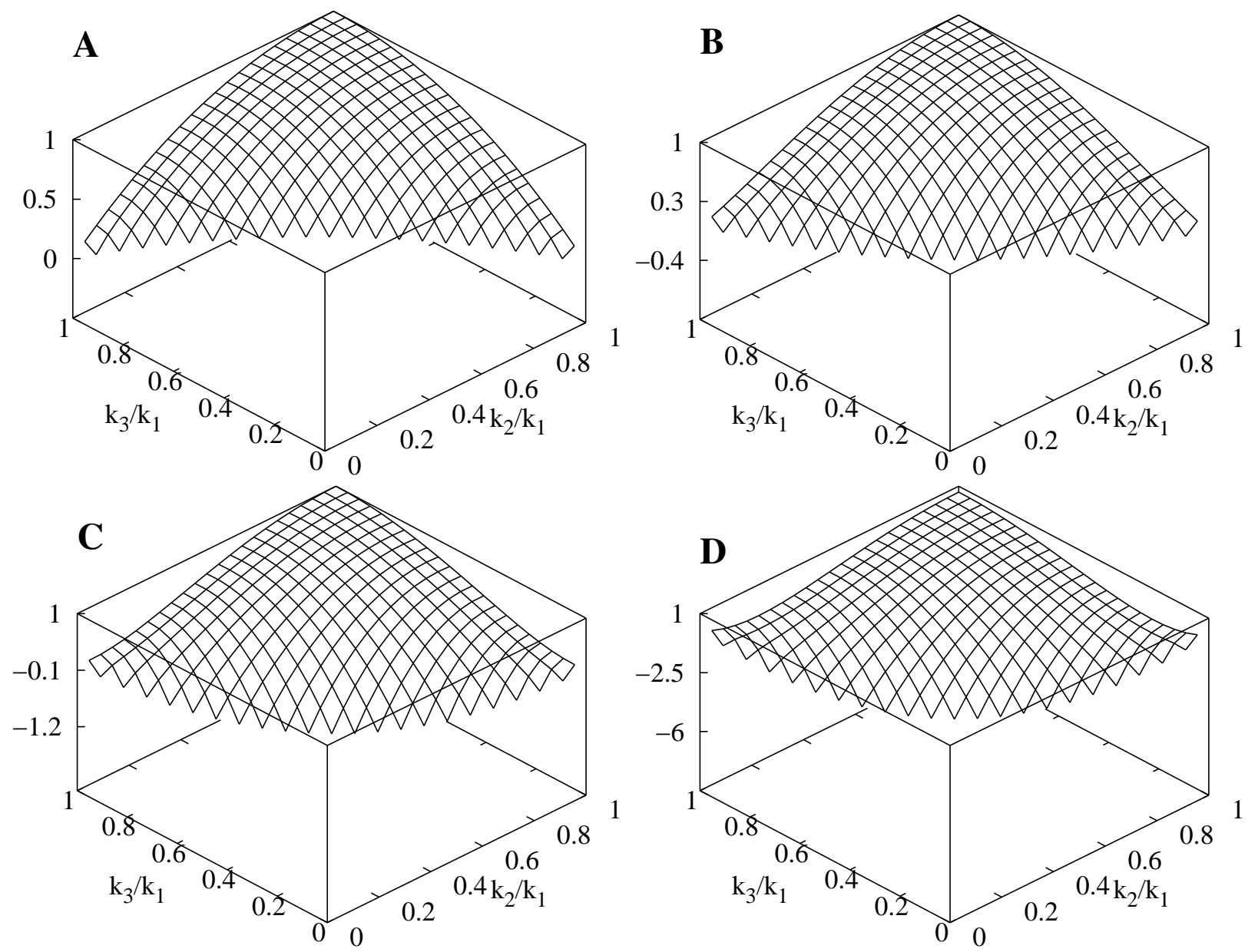

Figure 2: The leading-order shape function $\mathcal{F}_{\mathcal{R}}^{\text {lead }}\left(1, k_{2} / k_{1}, k_{3} / k_{1}\right)\left(k_{2} / k_{1}\right)^{2}\left(k_{3} / k_{1}\right)^{2}$ for $c_{s}^{2}=2.0 \times 10^{-3}$ normalized to 1 at the equilateral configuration. Each panel corresponds to the models A, B, C, D.

constraint on $c_{s}^{2}$ comes from the enfolded bound (4) rather than the equilateral bound (2), i.e., $c_{s}^{2}>3.6 \times 10^{-4}$. Note that the scalar spectral index is $n_{\mathcal{R}}=1$ and that the same relation as Eq. (132) holds in Model C. Hence the upper bound of $c_{s}^{2}$ coming from the observational constraint of $r$ is the same as that of Model B, i.e., $c_{s}^{2}<1.6 \times 10^{-2}$ for $r<0.38$ and $c_{s}^{2}<7.7 \times 10^{-3}$ for $r=0.13$.

In Fig. 2 we plot the shape of non-Gaussianities for $c_{s}^{2}=2.0 \times 10^{-3}$, in which case the shape is between the equilateral and orthogonal ones shown in Fig. 1 In fact, we have $C^{\text {equil }}=0.58145, C^{\text {ortho }}=0.75322$, and $C^{\text {enfold }}=-0.33691$ for $c_{s}^{2}=2.0 \times 10^{-3}$. The orthogonal contribution tends to be less important for the values of $c_{s}^{2}$ larger than the order of $10^{-2}$.

\section{D. k-inflation with the term $G_{5}(X)$}

Finally we study the following model (dubbed Model D) [62]

$$
P(X)=-X+\frac{X^{2}}{2 M^{4}}, \quad G_{5}(X)=\frac{\mu X^{2}}{M^{10}} .
$$

In this case, for $x=X / M^{4}$ close to 1 , there is a de Sitter solution satisfying the conditions $H^{2}=M^{4} /\left(6 M_{\mathrm{pl}}^{2}\right)$ and $\mu^{2} M^{4} / M_{\mathrm{pl}}^{6}=27(1-x)^{2} / 25$. Since $\delta \mathcal{C}_{6}=3 \delta \mathcal{C}_{7}=36(1-x) / 5, \epsilon_{s}=18(1-x) / 5, c_{s}^{2}=3(1-x) / 10$, and $\lambda / \Sigma=1 / 2$, the bispectrum is given by

$$
\mathcal{A}_{\mathcal{R}}^{\text {lead }}=\frac{13}{12}\left[-\frac{1}{c_{s}^{2}}\left(\frac{\beta}{8}-\frac{5}{36}\right)-\frac{1}{2}+\beta\right] S_{7}^{\text {equil }}-\frac{14-13 \beta}{12}\left(\frac{1}{8 c_{s}^{2}}-1\right) S_{7}^{\text {ortho }} .
$$




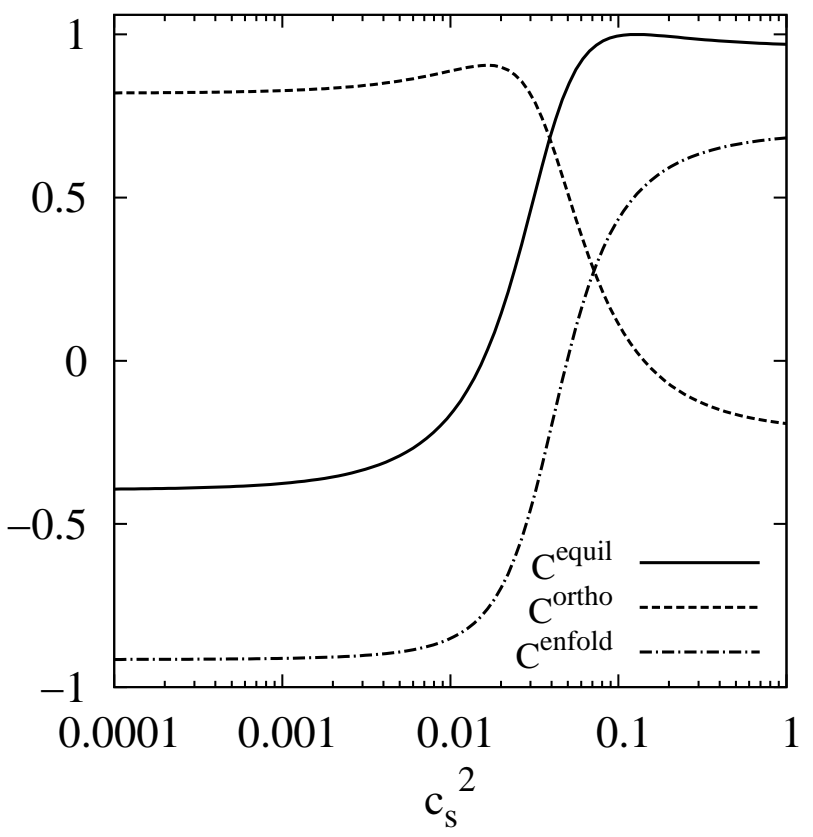

Figure 3: The correlations (120)-(122) versus $c_{s}^{2}$ for Model D. In the limit that $c_{s}^{2} \ll 1,\left|C^{\text {enfold }}\right|$ and $\left|C^{\text {ortho }}\right|$ are larger than $\left|C^{\text {equil }}\right|$. On the other hand, for $c_{s}^{2} \gtrsim 0.04,\left|C^{\text {equil }}\right|$ is largest.

In the limit $c_{s}^{2} \ll 1$ we have $\mathcal{A}_{\mathcal{R}}^{\text {lead }} \simeq\left(-0.012 / c_{s}^{2}\right) S_{7}^{\text {equil }}+\left(0.016 / c_{s}^{2}\right) S_{7}^{\text {ortho }}=-\left(0.023 / c_{s}^{2}\right) S_{7}^{\text {enfold }}+\left(0.005 / c_{s}^{2}\right) S_{7}^{\text {ortho }}$, in which case the sign in front of the shape $S_{7}^{\text {equil }}$ in Eq. (137) is opposite to those in the models B and C. In the same limit the equilateral and enfolded non-linear parameters are $f_{\mathrm{NL}}^{\text {equil,lead }} \simeq 5 /\left(972 c_{s}^{2}\right)$ and $f_{\mathrm{NL}}^{\text {enfold,lead }} \simeq-1 /\left(32 c_{s}^{2}\right)$, respectively. In this case $f_{\mathrm{NL}}^{\text {equil,lead }}$ is smaller than $\left|f_{\mathrm{NL}}^{\text {enfold,lead }}\right|$ by one order of magnitude. The WMAP 9 year enfolded bound (41) gives the constraint $c_{s}^{2}>3.6 \times 10^{-4}$. In Model D we have that $n_{\mathcal{R}}=1$ and that the relation between $r$ and $c_{s}^{2}$ is the same as Eq. (132). Hence the upper bound on $c_{s}^{2}$ is the same as that of Models B and C.

The shape of non-Gaussianities for $c_{s}^{2}=2.0 \times 10^{-3}$ is plotted in Fig. 2. When $c_{s}^{2}=2.0 \times 10^{-3}$ the correlations (120)-(122) are $C^{\text {equil }}=-0.35587, C^{\text {ortho }}=0.83545$, and $C^{\text {enfold }}=-0.90787$, respectively, in which case the shape has quite high (anti)-correlations with both the orthogonal and enfolded templates. In Fig. 3 we show the correlations $C^{\text {equil }}, C^{\text {ortho }}$, and $C^{\text {enfold }}$ versus $c_{s}^{2}$. For $c_{s}^{2} \gtrsim 0.04$ the correlation with the equilateral template is larger than those with other templates. For $c_{s}^{2} \lesssim 0.04$ the contributions of the orthogonal and enfolded shapes tend to be important. In the limit that $c_{s}^{2} \ll 1,\left|C^{\text {enfold }}\right|$ is largest among other correlations. Model D is an explicit example where the orthogonal (or enfolded) shape provides a significant contribution to the bispectrum.

\section{CONCLUSIONS}

In the Horndeski's most general scalar-tensor theories we derived the three-point correlation function of primordial curvature perturbations generated during inflation in the presence of slow-variation corrections to the leading-order bispectrum. Unlike previous works [61, 62], the bispectrum (84) is valid for any shape of non-Gaussianities at first order of $\epsilon$.

In the squeezed limit $\left(k_{3} \rightarrow 0, k_{1} \rightarrow k_{2}\right)$ the leading-order bispectrum (85) vanishes, so that the correction (86) is the dominant contribution to $\mathcal{A}_{\mathcal{R}}$. By using Eq. (86), we showed that the non-linear parameter in this limit is given by $f_{\mathrm{NL}}^{\text {local }}=(5 / 12)\left(1-n_{\mathcal{R}}\right)$. This agrees with the result of Refs. [12, 47] in which the three-point correlation function was derived by dealing with the long-wavelength curvature perturbation (mode $\boldsymbol{k}_{3}$ ) as a classical background. As demonstrated in Ref. [47], this result should be valid for any single-field inflation in which the decaying mode of $\mathcal{R}$ is neglected relative to the growing mode. Our direct computation of the three-point correlation function in the presence of all possible slow-variation corrections is another independent proof that the non-Gaussianity consistency relation holds for most general single-field theories with second-order equations of motion.

The result of the local non-Gaussianities shows that $\left|f_{\mathrm{NL}}^{\text {local }}\right|$ is much smaller than 1 , e.g., $f_{\mathrm{NL}}^{\text {local }}=0.0125$ for $n_{\mathcal{R}}=0.97$. In the case where the shape of non-Gaussianities is not exactly the squeezed one $\left(0<r_{3}=k_{3} / k_{1} \ll 1\right)$, the leading-order bispectrum gives the non-linear parameter $\left|f_{\mathrm{NL}}^{\text {lead }}\right| \approx r_{3}^{2} / c_{s}^{2}$. Hence the leading-order term dominates 
over the correction for $r_{3}>c_{s} \sqrt{1-n_{\mathcal{R}}}$. For the models with $c_{s}^{2} \ll 1$ the non-linear parameter can be as large as $\left|f_{\mathrm{NL}}\right|>1$ with the growth of $r_{3}$. By measuring the shape which is not so squeezed, it should be possible to discriminate the models with different values of $c_{s}^{2}$. The leading-order non-linear parameters in the equilateral and enfolded limits are given by Eqs. (97) and (101), respectively, whose magnitudes can be larger than 1 for the models with $c_{s}^{2} \ll 1$. These results will be useful to constrain concrete models of inflation in future high-precision observations.

We also showed that the leading-order bispectrum can be expressed in terms of the sum of the two bases $S_{7}^{\text {equil }}$ and $S_{7}^{\text {ortho }}$. The shape $S_{7}^{\text {equil }}$ is very highly correlated with the equilateral template (109). It also vanishes in both local and enfolded limits. The shape $S_{7}^{\text {ortho }}$, which is defined by (113), is exactly orthogonal to $S_{7}^{\text {equil }}$. The coefficients $c_{1}$

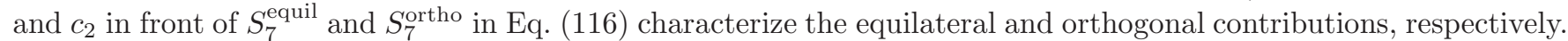

In Sec. VI we presented concrete models in which the orthogonal shape can provide important contributions to the bispectrum. In power-law k-inflation the shape of non-Gaussianities is well approximated by the equilateral type. However, in k-inflation described by the Lagrangian $P(X)=-X+X^{2} /\left(2 M^{4}\right)$ with a number of different Galileon terms like $G_{4}(X)=\mu X^{2} / M^{7}$ and $G_{5}(X)=\mu X^{2} / M^{10}$, we found that the orthogonal contribution is crucially important for $c_{s}^{2} \ll 1$. In the presence of the term $G_{5}(X)=\mu X^{2} / M^{10}$, the correlations with the orthogonal and enfolded templates in the regime $c_{s}^{2} \ll 1$ are higher than that with the equilateral template.

It will be interesting to see how the observations such as Planck [75] provide the constraints on the scalar nonGaussianities as well as the scalar spectral index and the tensor-to-scalar ratio. In particular, if future observations confirm the value $\left|f_{\mathrm{NL}}^{\text {local }}\right|>1$ at more than $95 \% \mathrm{CL}$, this implies that we need to go beyond the slow-variation singlefield inflationary scenario (including the Horndeski's theories). The information of other shapes of non-Gaussianities (including the not so squeezed one) will be useful to discriminate between many different models.

\section{ACKNOWLEDGEMENTS}

We are grateful to Eiichiro Komatsu for motivating us to derive the full bispectrum with slow-variation corrections in the Horndeski's theories. We also thank Xingang Chen, Shinji Mukohyama, and Misao Sasaki for useful correspondence and discussions. This work was supported in part by the Grant-in-Aid for Scientific Research Fund of No. 24540286 and Scientific Research on Innovative Areas (No. 21111006).

\section{Appendix A: Corrections to the bispectrum}

Following the calculations of Ref. [56] we present the explicit forms of the corrections to the bispectrum (56). They come from the first three integrals in the action (50). We write each $\mathcal{O}(\epsilon)$ contribution to the bispectrum as $\Delta \mathcal{A}_{\mathcal{R}}^{(i)}$ $(i=1,2,3)$. Note that similar calculations were also carried out in Ref. 31].

(i) $\int d t d^{3} x a^{3} \tilde{\mathcal{C}}_{1} M_{\mathrm{pl}}^{2} \mathcal{R} \dot{\mathcal{R}}^{2}$

- (a) The correction from the variation of $\tilde{\mathcal{C}}_{1}$ in Eq. (700)

$$
\Delta \mathcal{A}_{\mathcal{R}}^{(1)} \supset-\left(\frac{c_{s}^{2}}{4 \epsilon_{s} F} \frac{1}{H} \frac{d \tilde{\mathcal{C}}_{1}^{\text {lead }}}{d t}\right)_{K}\left[\left(1-2 \gamma_{1}\right) \frac{1}{K} \sum_{i>j} k_{i}^{2} k_{j}^{2}-\left(1-\gamma_{1}\right) \frac{1}{K^{2}} \sum_{i \neq j} k_{i}^{2} k_{j}^{3}\right] .
$$

- (b) The correction from the scale factor $a$ in Eq. (71)

$$
\Delta \mathcal{A}_{\mathcal{R}}^{(1)} \supset\left(\frac{c_{s}^{2} \epsilon}{2 \epsilon_{s} F} \tilde{\mathcal{C}}_{1}^{\text {lead }}\right)_{K}\left[\left(1+2 \gamma_{1}\right) \frac{1}{K} \sum_{i>j} k_{i}^{2} k_{j}^{2}-\gamma_{1} \frac{1}{K^{2}} \sum_{i \neq j} k_{i}^{2} k_{j}^{3}\right] .
$$

- (c) The contribution from the correction to $u\left(0, k_{i}\right)$ in Eq. (73)

$$
\begin{aligned}
\Delta \mathcal{A}_{\mathcal{R}}^{(1)} \supset & -\left(\frac{c_{s}^{2}}{4 \epsilon_{s} F} \tilde{\mathcal{C}}_{1}^{\text {lead }}\right)_{K}\left[3\left(1+\gamma_{2}\right) \epsilon+\frac{3 \gamma_{2}}{2} \eta_{s F}+3\left(1+\frac{\gamma_{2}}{2}\right) s+\left(\epsilon+\frac{\eta_{s F}}{2}+\frac{s}{2}\right) \ln \frac{k_{1} k_{2} k_{3}}{K^{3}}\right] \\
& \times\left(\frac{2}{K} \sum_{i>j} k_{i}^{2} k_{j}^{2}-\frac{1}{K^{2}} \sum_{i \neq j} k_{i}^{2} k_{j}^{3}\right) .
\end{aligned}
$$


- (d) The contribution from the correction to $u^{*}\left(\tau, k_{i}\right)$ in Eq. (75)

$$
\begin{aligned}
\Delta \mathcal{A}_{\mathcal{R}}^{(1)} \supset & \left(\frac{c_{s}^{2}}{4 \epsilon_{s} F} \tilde{\mathcal{C}}_{1}^{\text {lead }}\right)_{K}\left[3\left(1-2 \gamma_{1}\right) s \frac{\left(k_{1} k_{2} k_{3}\right)^{2}}{K^{3}}-\left\{\left(1+2 \gamma_{1}\right) \epsilon+\left(\gamma_{1}-\frac{1}{2}\right) \eta_{s F}+\left(\frac{3}{2}+\gamma_{1}\right) s\right\} \frac{1}{K} \sum_{i>j} k_{i}^{2} k_{j}^{2}\right. \\
& \left.+\left\{\gamma_{1} \epsilon-\frac{1}{2}\left(1-\gamma_{1}\right) \eta_{s F}+\frac{1}{2}\left(1+\gamma_{1}\right) s\right\} \frac{1}{K^{2}} \sum_{i \neq j} k_{i}^{2} k_{j}^{3}+\left(\epsilon+\frac{\eta_{s F}}{2}+\frac{s}{2}\right)\left(\frac{k_{2}^{2} k_{3}^{2}}{k_{1}} \mathcal{G}+\text { perm. }\right)\right],
\end{aligned}
$$

where

$$
\mathcal{G}\left(k_{1}, k_{2}, k_{3}\right) \equiv \operatorname{Re}\left[\int_{0}^{\infty} d x_{1} h^{*}\left(x_{1}\right) e^{-\frac{k_{2}+k_{3}}{k_{1}} x_{1}}\right]
$$

and $x_{1}=-k_{1} c_{s K} \tau$.

- (e) The contribution from the correction to $\frac{d}{d \tau} u^{*}\left(\tau, k_{i}\right)$ in Eq. (76)

$$
\begin{aligned}
\Delta \mathcal{A}_{\mathcal{R}}^{(1)} \supset & -\left(\frac{c_{s}^{2}}{4 \epsilon_{s} F} \tilde{\mathcal{C}}_{1}^{\text {lead }}\right)_{K}\left[\left\{2\left(1+2 \gamma_{1}\right) \epsilon+\left(2 \gamma_{1}-1\right)\left(\eta_{s F}-3 s\right)\right\} \frac{1}{K} \sum_{i>j} k_{i}^{2} k_{j}^{2}+\left(2 \epsilon+\eta_{s F}+s\right) k_{1} k_{2} k_{3}\right. \\
& -\left\{2 \gamma_{1} \epsilon+\left(\gamma_{1}-1\right) \eta_{s F}+\left(4-6 \gamma_{1}\right) s\right\} \frac{1}{K^{2}} \sum_{i \neq j} k_{i}^{2} k_{j}^{3}+\left(1-2 \gamma_{1}\right) s\left(\frac{1}{K^{3}} \sum_{i \neq j} k_{i}^{2} k_{j}^{4}+\frac{2}{K^{3}} \sum_{i>j} k_{i}^{3} k_{j}^{3}\right) \\
& \left.-\left(2 \epsilon+\eta_{s F}+s\right)\left\{\sum_{i} k_{i}^{3}+\sum_{i \neq j} k_{i} k_{j}^{2}+\sum_{i} k_{i}^{3} \operatorname{Re} \int_{0}^{\infty} d x_{K} \frac{e^{-i x_{K}}}{x_{K}}-\frac{1}{2}(\mathcal{M}+\text { perm. })\right\}\right],
\end{aligned}
$$

where $x_{K}=-K c_{s K} \tau$, and

$$
\mathcal{M}\left(k_{1}, k_{2}, k_{3}\right) \equiv-k_{1} \operatorname{Re}\left[\int_{0}^{\infty} d x_{1} \frac{1}{x_{1}}\left(k_{2}^{2}+k_{3}^{2}+i k_{2} k_{3} \frac{k_{2}+k_{3}}{k_{1}} x_{1}\right) e^{-i \frac{k_{2}+k_{3}}{k_{1}} x_{1}} \frac{d h^{*}\left(x_{1}\right)}{d x_{1}}\right] .
$$

(ii) $\int d t d^{3} x a \tilde{\mathcal{C}}_{2} M_{\mathrm{pl}}^{2} \mathcal{R}(\partial \mathcal{R})^{2}$

- (a) The correction from the variation of $\tilde{\mathcal{C}}_{2}$

$$
\Delta \mathcal{A}_{\mathcal{R}}^{(2)} \supset-\left(\frac{1}{4 \epsilon_{s} F} \frac{1}{H} \frac{d \tilde{\mathcal{C}}_{2}^{\text {lead }}}{d t}\right)_{K}\left[\left(1-\gamma_{1}\right)\left(\frac{1}{2} \sum_{i} k_{i}^{3}-\frac{1}{K^{2}} \sum_{i \neq j} k_{i}^{2} k_{j}^{3}\right)-\frac{1}{2} k_{1} k_{2} k_{3}+\frac{1-2 \gamma_{1}}{K} \sum_{i>j} k_{i}^{2} k_{j}^{2}+\frac{1}{2} \sum_{i \neq j} k_{i} k_{j}^{2}\right] .
$$

- (b) The correction from the scale factor $a$

$$
\Delta \mathcal{A}_{\mathcal{R}}^{(2)} \supset\left(\frac{\epsilon}{2 \epsilon_{s} F} \tilde{\mathcal{C}}_{2}^{\text {lead }}\right)_{K}\left[\gamma_{1}\left(\frac{1}{2} \sum_{i} k_{i}^{3}-\frac{1}{K^{2}} \sum_{i \neq j} k_{i}^{2} k_{j}^{3}\right)+\frac{1}{2} k_{1} k_{2} k_{3}+\frac{1+2 \gamma_{1}}{K} \sum_{i>j} k_{i}^{2} k_{j}^{2}-\frac{1}{2} \sum_{i \neq j} k_{i} k_{j}^{2}\right] .
$$

- (c) The contribution from the correction to $u\left(0, k_{i}\right)$

$$
\begin{aligned}
\Delta \mathcal{A}_{\mathcal{R}}^{(2)} \supset & -\left(\frac{1}{4 \epsilon_{s} F} \tilde{\mathcal{C}}_{2}^{\text {lead }}\right)_{K}\left[3\left(1+\gamma_{2}\right) \epsilon+\frac{3 \gamma_{2}}{2} \eta_{s F}+3\left(1+\frac{\gamma_{2}}{2}\right) s+\left(\epsilon+\frac{\eta_{s F}}{2}+\frac{s}{2}\right) \ln \frac{k_{1} k_{2} k_{3}}{K^{3}}\right] \\
& \times\left(\frac{1}{2} \sum_{i} k_{i}^{3}+\frac{2}{K} \sum_{i>j} k_{i}^{2} k_{j}^{2}-\frac{1}{K^{2}} \sum_{i \neq j} k_{i}^{2} k_{j}^{3}\right) .
\end{aligned}
$$


- (d) The contribution from the correction to $u^{*}\left(\tau, k_{i}\right)$

$$
\begin{aligned}
\Delta \mathcal{A}_{\mathcal{R}}^{(2)} \supset & -\left(\frac{1}{8 \epsilon_{s} F} \tilde{\mathcal{C}}_{2}^{\text {lead }}\right)_{K}\left[\left\{3 \gamma_{1} \epsilon+\frac{3}{2}\left(\gamma_{1}-1\right) \eta_{s F}+\frac{3}{2}\left(1+\gamma_{1}\right) s\right\} \sum_{i} k_{i}^{3}-3\left(\epsilon+\frac{\eta_{s F}}{2}+\frac{s}{2}\right) \sum_{i \neq j} k_{i} k_{j}^{2}\right. \\
& +\left\{3 \epsilon+\frac{3}{2} \eta_{s F}+\left(\frac{5}{2}-2 \gamma_{1}\right) s\right\} k_{1} k_{2} k_{3}+\left\{6\left(1+2 \gamma_{1}\right) \epsilon-3\left(1-2 \gamma_{1}\right) \eta_{s F}+\left(5+6 \gamma_{1}\right) s\right\} \frac{1}{K} \sum_{i>j} k_{i}^{2} k_{j}^{2} \\
& -\left\{6 \gamma_{1} \epsilon+3\left(\gamma_{1}-1\right) \eta_{s F}+\left(1+6 \gamma_{1}\right) s\right\} \frac{1}{K^{2}} \sum_{i \neq j} k_{i}^{2} k_{j}^{3}-\left(1+\gamma_{1}\right) s \frac{1}{K} \sum_{i} k_{i}^{4}-\gamma_{1} s \frac{1}{K^{2}} \sum_{i \neq j} k_{i} k_{j}^{4} \\
& \left.+\left(2 \epsilon+\eta_{s F}+s\right)(\mathcal{N}+\text { perm. })\right]
\end{aligned}
$$

where

$$
\mathcal{N}\left(k_{1}, k_{2}, k_{3}\right) \equiv \frac{k_{1}}{2} \sum_{i} k_{i}^{2} \operatorname{Re}\left[\int_{0}^{\infty} d x_{1} \frac{1}{x_{1}^{2}} e^{-i x_{1} \frac{k_{2}+k_{3}}{k_{1}}}\left(-1-i \frac{k_{2}+k_{3}}{k_{1}} x_{1}+\frac{k_{2} k_{3}}{k_{1}^{2}} x_{1}^{2}\right) h^{*}\left(x_{1}\right)\right] .
$$

Note that the coefficient $\tilde{\mathcal{C}}_{2}^{\text {lead }}$ is related to $\tilde{\mathcal{C}}_{1}^{\text {lead }}$ via $\tilde{\mathcal{C}}_{2}^{\text {lead }}=-\left(c_{s}^{2} / 3\right) \tilde{\mathcal{C}}_{1}^{\text {lead }}$.

(iii) $\int d t d^{3} x a^{3} \tilde{\mathcal{C}}_{3} M_{\mathrm{pl}} \dot{\mathcal{R}}^{3}$

- (a) The correction from the variation of $\tilde{\mathcal{C}}_{3}$

$$
\Delta \mathcal{A}_{\mathcal{R}}^{(3)} \supset\left(\frac{3\left(2 \gamma_{1}-3\right) c_{s}^{2}}{4 \epsilon_{s} F M_{\mathrm{pl}}} \frac{d \tilde{\mathcal{C}}_{3}^{\text {lead }}}{d t}\right)_{K} \frac{\left(k_{1} k_{2} k_{3}\right)^{2}}{K^{3}} .
$$

- (b) The correction from the scale factor $a$

$$
\Delta \mathcal{A}_{\mathcal{R}}^{(3)} \supset\left(\frac{3\left(2 \gamma_{1}-1\right) c_{s}^{2} \epsilon H}{4 \epsilon_{s} F M_{\mathrm{pl}}} \tilde{\mathcal{C}}_{3}^{\text {lead }}\right)_{K} \frac{\left(k_{1} k_{2} k_{3}\right)^{2}}{K^{3}} .
$$

- (c) The contribution from the correction to $u\left(0, k_{i}\right)$

$$
\Delta \mathcal{A}_{\mathcal{R}}^{(3)} \supset-\left(\frac{3 H c_{s}^{2}}{2 \epsilon_{s} F M_{\mathrm{pl}}} \tilde{\mathcal{C}}_{3}^{\text {lead }}\right)_{K}\left[3\left(1+\gamma_{2}\right) \epsilon+\frac{3}{2} \gamma_{2} \eta_{s F}+3\left(1+\frac{\gamma_{2}}{2}\right) s+\left(\epsilon+\frac{\eta_{s F}}{2}+\frac{s}{2}\right) \ln \frac{k_{1} k_{2} k_{3}}{K^{3}}\right] \frac{\left(k_{1} k_{2} k_{3}\right)^{2}}{K^{3}} .
$$

- (d) The contribution from the correction to $\frac{d}{d \tau} u^{*}\left(\tau, k_{i}\right)$

$$
\begin{aligned}
& \Delta \mathcal{A}_{\mathcal{R}}^{(3)} \supset\left(\frac{3 H c_{s}^{2}}{4 \epsilon_{s} F M_{\mathrm{pl}}} \tilde{\mathcal{C}}_{3}^{\text {lead }}\right)_{K}\left[\left\{3\left(1-2 \gamma_{1}\right) \epsilon+3\left(\frac{3}{2}-\gamma_{1}\right) \eta_{s F}+\left(3 \gamma_{1}-\frac{17}{2}\right) s\right\} \frac{\left(k_{1} k_{2} k_{3}\right)^{2}}{K^{3}}\right. \\
&\left.+\left(\epsilon+\frac{\eta_{s F}}{2}+\frac{s}{2}\right)\left\{\left(\frac{1}{K^{2}} \sum_{i \neq j} k_{i}^{2} k_{j}^{3}-\frac{2}{K} \sum_{i>j} k_{i}^{2} k_{j}^{2}\right)+\mathcal{U}\right\}\right],
\end{aligned}
$$

where $\mathcal{U}$ is defined in Eq. (82).

\section{Appendix B: Expressions for $\mathcal{U}$ and $\mathcal{V}$}

We evaluate the integrals $\mathcal{U}$ and $\mathcal{V}$ defined in Eqs. (82) and (81) for general values of $r_{2}=k_{2} / k_{1}, r_{3}=k_{3} / k_{1}$, and in three different limits: local shapes $\left(r_{3} \rightarrow 0, r_{2}=1\right)$, equilateral $\left(r_{3}=1, r_{2}=1\right)$, and enfolded $\left(r_{3} \rightarrow 1 / 2, r_{2} \rightarrow 1 / 2\right)$ shapes. In doing so, we need to use the following relations

$$
\begin{aligned}
h^{*}(x)= & 2 \sin (x)+[\sin (x)-x \cos (x)] \operatorname{Ci}(2 x)-[\cos (x)+x \sin (x)] \operatorname{Si}(2 x) \\
& +i\{2 \cos (x)+\pi \sin (x)-\pi x \cos (x)-[\cos (x)+x \sin (x)] \operatorname{Ci}(2 x)-[\sin (x)-x \cos (x)] \operatorname{Si}(2 x)\}, \\
\frac{d h^{*}(x)}{d x}= & \cos (x)-\sin (x) / x+x \sin (x) \operatorname{Ci}(2 x)-x \cos (x) \operatorname{Si}(2 x) \\
& +i[\pi x \sin (x)-\sin (x)-\cos (x) / x-x \cos (x) \operatorname{Ci}(2 x)-x \sin (x) \operatorname{Si}(2 x)] .
\end{aligned}
$$




\section{The $\mathcal{U}$ integral}

Let us first evaluate the integral $\mathcal{U}$. We employ a few different procedures of regularizations, but they lead to the same final results. One possibility is to solve directly the integral, as

$$
\mathcal{U}=\int_{0}^{\infty} f_{\mathcal{U}}\left(x_{1}\right) d x_{1}+\text { perm. }=\lim _{y \rightarrow \infty} \int_{0}^{y} f_{\mathcal{U}}\left(x_{1}\right) d x_{1}+\text { perm. }
$$

where $f_{\mathcal{U}}$ is the integrand in Eq. (82). We can either neglect the terms which rapidly oscillate around 0 , or we can, equivalently, shift $y$ in the complex domain for the oscillating terms like $e^{ \pm i k y} \rightarrow e^{ \pm i k \rho^{2}(1 \pm i / \rho)} \rightarrow 0($ Method I). Another possible method (Method II) consists of solving the following limit

$$
\mathcal{U}=\int_{0}^{\infty} f_{\mathcal{U}}\left(x_{1}\right) d x_{1}+\text { perm. }=\lim _{c \rightarrow 0} \int_{0}^{\infty} e^{-c x_{1}} f_{\mathcal{U}}\left(x_{1}\right) d x_{1}+\text { perm. } .
$$

We need to check whether both methods lead to the same results, and we can - at least for some values of $r_{2}, r_{3}$ this proves to be possible - check the result against numerical integrations.

By employing the Method I mentioned above, the result of the integration is

$$
\begin{aligned}
\mathcal{U}\left(r_{2}, r_{3}\right)= & k_{1}^{3}\left[\frac{4 r_{2}^{2} r_{3}^{3}\left(3 r_{2}^{2}-6 r_{2}+r_{3}^{2}+3\right)}{\left(r_{2}-r_{3}-1\right)^{3}\left(r_{2}+r_{3}-1\right)^{3}}-\frac{2 r_{2}^{2} r_{3}^{2}}{\left(r_{2}-r_{3}+1\right)^{3}}\right] \ln 2 \\
& +\frac{k_{1}^{3}}{\left(r_{2}+r_{3}+1\right)^{2}}\left\{\frac{\left[\left(r_{2}+1\right)^{3}-6 r_{3}^{2}\left(r_{2}+1\right)+r_{3}^{3}\right] r_{2}^{2}}{\left(r_{2}-r_{3}+1\right)^{2}}+\frac{r_{3}^{2}\left[r_{2}^{3}+3 r_{3} r_{2}^{2}+3\left(r_{3}^{2}-2\right) r_{2}+r_{3}^{3}-6 r_{3}+1\right] r_{2}^{2}}{\left(r_{2}+r_{3}-1\right)^{2}}\right. \\
& +\frac{r_{3}^{2}\left[r_{2}^{3}-6\left(r_{3}+1\right) r_{2}^{2}+\left(r_{3}+1\right)^{3}\right]}{\left(1-r_{2}+r_{3}\right)^{2}}+\frac{2 r_{3}^{2}\left(r_{2}+r_{3}+1\right)^{2} r_{2}^{2}}{\left(r_{2}-r_{3}+1\right)^{3}} \ln \left(\frac{r_{2}+r_{3}+1}{r_{3}}\right) \\
& \left.+\frac{2 r_{3}^{2}\left(r_{2}+r_{3}+1\right)^{2} r_{2}^{2}}{\left(1-r_{2}+r_{3}\right)^{3}} \ln \left(\frac{r_{2}+r_{3}+1}{r_{2}}\right)+\frac{2 r_{3}^{2}\left(r_{2}+r_{3}+1\right)^{2} r_{2}^{2}}{\left(r_{2}+r_{3}-1\right)^{3}} \ln \left(r_{2}+r_{3}+1\right)\right\}
\end{aligned}
$$

where $\mathcal{U}\left(r_{1}, r_{2}\right)=\mathcal{U}\left(r_{2}, r_{1}\right)$, that is $\mathcal{U}$ is symmetric under the exchange $r_{1} \leftrightarrow r_{2}$.

Let us consider this general expression in several different cases. Some of these cases look - only apparently singular: this behavior takes place as the triangle of the momenta $\boldsymbol{k}_{i}$ degenerates into a line.

- Equilateral case, $r_{2}=r_{3}=1$. In this case we find

$$
\mathcal{U}(1,1)=\mathcal{U}_{\text {equil }}=[6 \ln (3 / 2)-1] k_{1}^{3} \approx 1.43279 k_{1}^{3}
$$

Since there is no apparent singular behavior for $\mathcal{U}$, this result can also be confirmed numerically ${ }^{6}$.

- Local case, $r_{2}=1, r_{3} \rightarrow 0$ (or $r_{2} \rightarrow 0, r_{3}=1$ ). In this case, the limit exists and gives

$$
\lim _{r_{3} \rightarrow 0} \mathcal{U}\left(1, r_{3}\right)=\mathcal{U}_{\text {local }}=\frac{1}{2} k_{1}^{3}
$$

The result in the local case matches with that derived by Chen et al. 56.

- Singular line, $r_{3}=\lim _{\epsilon \rightarrow 0}\left(1-r_{2}+\epsilon\right)$, with $0<r_{2}<1$. In this case we find

$$
\lim _{\epsilon \rightarrow 0} \mathcal{U}=\frac{r_{2}\left\{\left(r_{2}-1\right) r_{2}\left[20\left(r_{2}-1\right) r_{2}+9\right]+6\right\}\left(r_{2}-1\right)-6\left(r_{2}-1\right)^{3} \ln \left(1-r_{2}\right)+6 r_{2}^{3} \ln r_{2}}{24\left(r_{2}-1\right) r_{2}} k_{1}^{3} .
$$

The limits $r_{2} \rightarrow 0,1$ give again the local result, as expected.

- Enfolded case, $r_{2}=1 / 2, r_{3}=\lim _{\epsilon \rightarrow 0}(1 / 2+\epsilon)$. This is a particular case of the previous one. Then we find

$$
\lim _{\epsilon \rightarrow 0} \mathcal{U}=\mathcal{U}_{\text {enfold }}=\frac{1}{24}(5+6 \ln 2) k_{1}^{3} \approx 0.38162 k_{1}^{3} \text {. }
$$

\footnotetext{
${ }^{6}$ Mathematica, working in high precision, returns this same numerical value. The same value can also be found by applying the Method II mentioned above.
} 
- Singular line, $r_{3}=\lim _{\epsilon \rightarrow 0}\left(r_{2}-1+\epsilon\right)$, with $r_{2}>1$. In this case we obtain

$$
\lim _{\epsilon \rightarrow 0} \mathcal{U}=\frac{\left(r_{2}-1\right)\left\{r_{2}\left[r_{2}\left(6 r_{2}^{2}-9 r_{2}+29\right)-40\right]+20\right\}+6 r_{2}^{3}\left[\left(r_{2}-1\right)^{3} \ln \left(\frac{r_{2}}{r_{2}-1}\right)+\ln r_{2}\right]}{24\left(r_{2}-1\right) r_{2}} k_{1}^{3} .
$$

In the limit $r_{2} \rightarrow 1^{+}$, this reproduces the local limit result, as expected.

- Singular line, $r_{3}=\lim _{\epsilon \rightarrow 0}\left(1+r_{2}-\epsilon\right)$. In this case we find

$$
\lim _{\epsilon \rightarrow 0} \mathcal{U}=\frac{r_{2}\left\{r_{2}\left\{r_{2}\left[3 r_{2}\left(2 r_{2}+5\right)+38\right]+15\right\}+6\right\}+6 r_{2}^{3}\left(r_{2}+1\right)^{3} \ln \left(\frac{1}{r_{2}}+1\right)+6\left(r_{2}+1\right)^{3} \ln \left(r_{2}+1\right)}{24 r_{2}\left(r_{2}+1\right)} k_{1}^{3},
$$

which recovers the local limit as $r_{2}=\epsilon \rightarrow 0$.

Therefore, we have shown that the physical limits are all finite.

\section{The $\mathcal{V}$ integral}

The next step is to compute the integral (81). It is convenient to solve the integral by studying the limit

$$
\mathcal{V}=\lim _{\varepsilon \rightarrow 0} \lim _{y \rightarrow \infty} \int_{\varepsilon}^{y} f_{\mathcal{V}}\left(x_{1}\right) d x_{1}+\text { perm. }
$$

where $f_{\mathcal{V}}$ is the integrand in Eq. (81). As for the $y \rightarrow \infty$ limit, we set the rapidly oscillating functions to vanish by regularizing $e^{ \pm i k y}$ as in the Method I of the integral $\mathcal{U}$. After taking the limit $\varepsilon \rightarrow 0$, we finally obtain

$$
\begin{aligned}
\mathcal{V}= & \frac{k_{1}^{3}}{3\left(r_{2}-r_{3}-1\right)\left(r_{2}+r_{3}-1\right)\left(r_{2}+r_{3}+1\right)}\left[3 r_{2}^{6}+6 r_{3} r_{2}^{5}+r_{2}^{4}\left(r_{3}^{2}-9 r_{3}-3\right)+r_{3} r_{2}^{3}\left(6 r_{3}^{2}-13 r_{3}+3\right)+3 r_{3}^{4} r_{2}^{2}+3\right. \\
& \left.-15 r_{3}^{3} r_{2}^{2}-4 r_{3}^{2} r_{2}^{2}+3 r_{2}^{2}\left(r_{3}-1\right)-12 r_{3}^{5}\left(r_{2}+1\right)-r_{2} r_{3}\left(19 r_{3}^{3}+15 r_{3}^{2}+13 r_{3}+9\right)-7 r_{3}^{6}+3 r_{3}^{4}+6 r_{3}^{3}+r_{3}^{2}+6 r_{3}\right] \\
& +\frac{k_{1}^{3}}{6\left(r_{2}-r_{3}+1\right)\left(r_{2}+r_{3}+1\right)}\left(7 r_{2}^{5}+5 r_{3} r_{2}^{4}+19 r_{2}^{4}-6 r_{3}^{2} r_{2}^{3}+5 r_{3} r_{2}^{3}+14 r_{2}^{3}-6 r_{3}^{3} r_{2}^{2}+6 r_{3}^{2} r_{2}^{2}+4 r_{3} r_{2}^{2}+14 r_{2}^{2}\right. \\
& \left.-r_{3}^{3} r_{2}+6 r_{3}^{2} r_{2}+5 r_{3} r_{2}+19 r_{2}+r_{3}^{5}-r_{3}^{4}-6 r_{3}^{3}-6 r_{3}^{2}+5 r_{3}+7-r_{3}^{4} r_{2}\right) \\
& -\frac{k_{1}^{3} \ln 2}{3\left(r_{2}-r_{3}-1\right)^{2}\left(r_{2}+r_{3}-1\right)^{2}} r_{3}^{3}\left(5 r_{2}^{4}-20 r_{2}^{3}-6 r_{3}^{2} r_{2}^{2}+22 r_{2}^{2}+4 r_{3}^{2} r_{2}-20 r_{2}+r_{3}^{4}-6 r_{3}^{2}+5\right) \\
& +\frac{k_{1}^{3} \ln \left(r_{2}+r_{3}+1\right)}{6\left(r_{2}+r_{3}-1\right)^{2}}\left(r_{2}^{5}+2 r_{3} r_{2}^{4}-2 r_{2}^{4}-3 r_{3}^{2} r_{2}^{3}-2 r_{3} r_{2}^{3}-3 r_{2}^{3}-3 r_{3}^{3} r_{2}^{2}+8 r_{3}^{2} r_{2}^{2}-8 r_{3} r_{2}^{2}+3 r_{2}^{2}+2 r_{3}^{4} r_{2}-2 r_{3}^{3} r_{2}\right. \\
& \left.-8 r_{3}^{2} r_{2}-2 r_{3} r_{2}+2 r_{2}+r_{3}^{5}-2 r_{3}^{4}-3 r_{3}^{3}+3 r_{3}^{2}+2 r_{3}-1\right) \\
& -\frac{k_{1}^{3} \ln \left(\frac{r_{2}+r_{3}+1}{r_{2}}\right)}{6\left(r_{2}-r_{3}-1\right)^{2}}\left(r_{2}^{5}-2 r_{3} r_{2}^{4}-2 r_{2}^{4}-3 r_{3}^{2} r_{2}^{3}+2 r_{3} r_{2}^{3}-3 r_{2}^{3}+3 r_{3}^{3} r_{2}^{2}+8 r_{3}^{2} r_{2}^{2}+8 r_{3} r_{2}^{2}+3 r_{2}^{2}+2 r_{3}^{4} r_{2}+2 r_{3}^{3} r_{2}\right. \\
& \left.-8 r_{3}^{2} r_{2}+2 r_{3} r_{2}+2 r_{2}-r_{3}^{5}-2 r_{3}^{4}+3 r_{3}^{3}+3 r_{3}^{2}-2 r_{3}-1\right) \\
& +\frac{k_{1}^{3} \ln \left(\frac{r_{2}+r_{3}+1}{2 r_{3}}\right)}{6\left(r_{2}-r_{3}+1\right)^{2}}\left(r_{2}^{5}-2 r_{3} r_{2}^{4}+2 r_{2}^{4}-3 r_{3}^{2} r_{2}^{3}-2 r_{3} r_{2}^{3}-3 r_{2}^{3}+3 r_{3}^{3} r_{2}^{2}-8 r_{3}^{2} r_{2}^{2}+8 r_{3} r_{2}^{2}-3 r_{2}^{2}+2 r_{3}^{4} r_{2}-2 r_{3}^{3} r_{2}\right. \\
& \left.-8 r_{3}^{2} r_{2}-2 r_{3} r_{2}+2 r_{2}-r_{3}^{5}+2 r_{3}^{4}+3 r_{3}^{3}-3 r_{3}^{2}-2 r_{3}+1\right) \\
(\mathrm{B} 13) &
\end{aligned}
$$

where $\mathcal{V}\left(r_{1}, r_{2}\right)=\mathcal{V}\left(r_{2}, r_{1}\right)$. Let us now analyze this expression, on the lines/points of physical interest.

- Equilateral case, $r_{2}=r_{3}=1$. In this case we find

$$
\mathcal{V}(1,1)=\mathcal{V}_{\text {equil }}=\frac{15}{2}[2+\ln (2 / 3)] k_{1}^{3} \approx 11.959 k_{1}^{3}
$$

- Local case, $r_{2}=1, r_{3} \rightarrow 0$ (or $r_{2} \rightarrow 0, r_{3}=1$ ). In this case, the limit exists and gives

$$
\lim _{r_{3} \rightarrow 0^{+}} \mathcal{V}\left(1, r_{3}\right)=\mathcal{V}_{\text {local }}=\frac{20}{3} k_{1}^{3},
$$

where this value of $\mathcal{V}_{\text {local }}$ matches with the one derived by Chen et al. [56]. 
- Singular line, $r_{3}=\lim _{\epsilon \rightarrow 0}\left(1-r_{2}+\epsilon\right)$, with $0<r_{2}<1$. In this case we obtain

$$
\lim _{\epsilon \rightarrow 0} \mathcal{V}=\frac{\left\{\left(r_{2}-1\right) r_{2}\left[11\left(r_{2}-1\right) r_{2}-105\right]-86\right\} r_{2}\left(1-r_{2}\right)+6\left(r_{2}-1\right)^{3} \ln \left(1-r_{2}\right)-6 r_{2}^{3} \ln r_{2}}{12\left(r_{2}-1\right) r_{2}} k_{1}^{3} .
$$

The limits $r_{2} \rightarrow 0,1$ again give the result (B15).

- Enfolded case, $r_{2}=1 / 2, r_{3}=\lim _{\epsilon \rightarrow 0}(1 / 2+\epsilon)$. This is a particular case of the previous one. Then we find

$$
\lim _{\epsilon \rightarrow 0} \mathcal{V}=\mathcal{V}_{\text {enfold }}=\left(\frac{315}{64}-\frac{1}{2} \ln 2\right) k_{1}^{3} \approx 4.5753 k_{1}^{3}
$$

- Singular line, $r_{3}=\lim _{\epsilon \rightarrow 0}\left(r_{2}-1+\epsilon\right)$, with $r_{2}>1$. In this case we find

$$
\lim _{\epsilon \rightarrow 0} \mathcal{V}=\frac{\left(r_{2}-1\right)\left[\left\{r_{2}\left[r_{2}\left(86 r_{2}-105\right)+94\right]+22\right\} r_{2}-6\left(r_{2}-1\right)^{2} r_{2}^{3} \ln \left(\frac{r_{2}}{r_{2}-1}\right)-11\right]-6 r_{2}^{3} \ln r_{2}}{12\left(r_{2}-1\right) r_{2}} k_{1}^{3} .
$$

Taking the limit $r_{2} \rightarrow 1^{+}$, we recover the result (B15).

- Singular line, $r_{3}=\lim _{\epsilon \rightarrow 0}\left(1+r_{2}-\epsilon\right)$. In this case we obtain

$$
\lim _{\epsilon \rightarrow 0} \mathcal{V}=\frac{r_{2}\left(r_{2}\left\{r_{2}\left[r_{2}\left(86 r_{2}+239\right)+295\right]+239\right\}+86\right)-6 r_{2}^{3}\left(r_{2}+1\right)^{3} \ln \left(\frac{1}{r_{2}}+1\right)-6\left(r_{2}+1\right)^{3} \ln \left(r_{2}+1\right)}{12 r_{2}\left(r_{2}+1\right)} k_{1}^{3},
$$

which again reproduces the value (B15) as $r_{2}=\epsilon \rightarrow 0$.

Therefore, the integral $\mathcal{V}$ remains finite in the physical parameter space of $r_{2}$ and $r_{3}$.

[1] A. A. Starobinsky, Phys. Lett. B 91, 99 (1980); D. Kazanas, Astrophys. J. 241 L59 (1980); K. Sato, Mon. Not. R. Astron. Soc. 195, 467 (1981); Phys. Lett. 99B, 66 (1981); A. H. Guth, Phys. Rev. D 23, 347 (1981).

[2] V. F. Mukhanov and G. V. Chibisov, JETP Lett. 33, 532 (1981); A. H. Guth and S. Y. Pi, Phys. Rev. Lett. 49 (1982) 1110; S. W. Hawking, Phys. Lett. B 115, 295 (1982); A. A. Starobinsky, Phys. Lett. B 117 (1982) 175; J. M. Bardeen, P. J. Steinhardt and M. S. Turner, Phys. Rev. D 28, 679 (1983).

[3] G. F. Smoot et al., Astrophys. J. 396, L1 (1992).

[4] D. N. Spergel et al. [WMAP Collaboration], Astrophys. J. Suppl. 148, 175 (2003) astro-ph/0302209].

[5] G. Hinshaw et al., arXiv:1212.5226 [astro-ph.CO].

[6] K. Enqvist and M. S. Sloth, Nucl. Phys. B 626, 395 (2002) hep-ph/0109214; D. H. Lyth and D. Wands, Phys. Lett. B 524, 5 (2002) hep-ph/0110002]; T. Moroi and T. Takahashi, Phys. Lett. B 522, 215 (2001) [Erratum-ibid. B 539, 303 (2002)] hep-ph/0110096.

[7] D. S. Salopek and J. R. Bond, Phys. Rev. D 42, 3936 (1990).

[8] A. Gangui, F. Lucchin, S. Matarrese and S. Mollerach, Astrophys. J. 430, 447 (1994).

[9] L. Verde, L. M. Wang, A. Heavens and M. Kamionkowski, Mon. Not. Roy. Astron. Soc. 313, L141 (2000) astro-ph/9906301.

[10] E. Komatsu and D. N. Spergel, Phys. Rev. D63, 063002 (2001).

[11] N. Bartolo, S. Matarrese, A. Riotto, Phys. Rev. D65, 103505 (2002) hep-ph/0112261; N. Bartolo, E. Komatsu, S. Matarrese and A. Riotto, Phys. Rept. 402, 103 (2004) astro-ph/0406398.

[12] J. M. Maldacena, JHEP 0305, 013 (2003) astro-ph/0210603.

[13] P. Creminelli, JCAP 0310, 003 (2003) astro-ph/0306122.

[14] G. I. Rigopoulos and E. P. S. Shellard, Phys. Rev. D 68, 123518 (2003) astro-ph/0306620; G. I. Rigopoulos and E. P. S. Shellard, JCAP 0510, 006 (2005) astro-ph/0405185.

[15] N. Arkani-Hamed, P. Creminelli, S. Mukohyama and M. Zaldarriaga, JCAP 0404, 001 (2004) hep-th/0312100.

[16] M. Alishahiha, E. Silverstein and D. Tong, Phys. Rev. D 70, 123505 (2004) hep-th/0404084.

[17] N. Bartolo, S. Matarrese and A. Riotto, Phys. Rev. D 69, 043503 (2004) hep-ph/0309033; M. Sasaki, J. Valiviita and D. Wands, Phys. Rev. D 74, 103003 (2006) astro-ph/0607627]; K. A. Malik and D. H. Lyth, JCAP 0609, 008 (2006) astro-ph/0604387.

[18] D. Seery and J. E. Lidsey, JCAP 0506, 003 (2005) astro-ph/0503692.

[19] D. Seery and J. E. Lidsey, JCAP 0509, 011 (2005) astro-ph/0506056. 
[20] D. H. Lyth and Y. Rodriguez, Phys. Rev. Lett. 95, 121302 (2005) astro-ph/0504045]; D. H. Lyth and Y. Rodriguez, Phys. Rev. D 71, 123508 (2005) astro-ph/0502578]; D. H. Lyth, K. A. Malik and M. Sasaki, JCAP 0505, 004 (2005) astro-ph/0411220.

[21] K. Enqvist and A. Vaihkonen, JCAP 0409, 006 (2004) hep-ph/0405103; B. A. Bassett, S. Tsujikawa and D. Wands, Rev. Mod. Phys. 78, 537 (2006) astro-ph/0507632; F. Vernizzi and D. Wands, JCAP 0605, 019 (2006); T. Battefeld and R. Easther, JCAP 0703, 020 (2007) astro-ph/0610296]; S. Yokoyama, T. Suyama and T. Tanaka, JCAP 0707, 013 (2007) arXiv:0705.3178 [astro-ph]]; F. Arroja, S. Mizuno and K. Koyama, JCAP 0808, 015 (2008) arXiv:0806.0619 [astro-ph]]; C. T. Byrnes, K. -Y. Choi and L. M. H. Hall, JCAP 0810, 008 (2008) arXiv:0807.1101 [astro-ph]]; C. T. Byrnes and G. Tasinato, JCAP 0908, 016 (2009) arXiv:0906.0767 [astro-ph.CO]]; H. R. S. Cogollo, Y. Rodriguez and C. A. ValenzuelaToledo, JCAP 0808 (2008) 029 arXiv:0806.1546 [astro-ph]]; C. T. Byrnes and K. -Y. Choi, Adv. Astron. 2010, 724525 (2010) arXiv:1002.3110 [astro-ph.CO]]; Y. Rodriguez and C. A. Valenzuela-Toledo, Phys. Rev. D 81 (2010) 023531 arXiv:0811.4092 [astro-ph]]; S. Renaux-Petel, S. Mizuno and K. Koyama, JCAP 1111, 042 (2011) arXiv:1108.0305 [astroph.CO]]; J. Elliston, D. J. Mulryne, D. Seery and R. Tavakol, JCAP 1111, 005 (2011) arXiv:1106.2153 [astro-ph.CO]].

[22] X. Chen, R. Easther and E. A. Lim, JCAP 0706, 023 (2007) astro-ph/0611645; X. Chen, R. Easther and E. A. Lim, JCAP 0804, 010 (2008) arXiv:0801.3295 [astro-ph]].

[23] N. Barnaby and J. M. Cline, JCAP 0707, 017 (2007) arXiv:0704.3426 [hep-th]]; N. Barnaby and J. M. Cline, JCAP 0806, 030 (2008) arXiv:0802.3218 [hep-th]].

[24] K. Koyama, S. Mizuno, F. Vernizzi and D. Wands, JCAP 0711, 024 (2007) arXiv:0708.4321 [hep-th]]; E. I. Buchbinder, J. Khoury and B. A. Ovrut, Phys. Rev. Lett. 100, 171302 (2008) arXiv:0710.5172 [hep-th]].

[25] T. Suyama and M. Yamaguchi, Phys. Rev. D 77, 023505 (2008) arXiv:0709.2545 [astro-ph]]; K. Ichikawa, T. Suyama, T. Takahashi and M. Yamaguchi, Phys. Rev. D 78, 023513 (2008) arXiv:0802.4138 [astro-ph]]; T. Suyama, T. Takahashi, M. Yamaguchi and S. Yokoyama, JCAP 1012, 030 (2010) arXiv:1009.1979 [astro-ph.CO]].

[26] D. Langlois, S. Renaux-Petel, D. A. Steer and T. Tanaka, Phys. Rev. Lett. 101, 061301 (2008) arXiv:0804.3139 [hep-th]]; D. Langlois, S. Renaux-Petel, D. A. Steer and T. Tanaka, Phys. Rev. D 78, 063523 (2008) arXiv:0806.0336 [hep-th]].

[27] M. Sasaki, Prog. Theor. Phys. 120, 159 (2008) arXiv:0805.0974 [astro-ph]]; A. Naruko and M. Sasaki, Prog. Theor. Phys. 121, 193 (2009) arXiv:0807.0180 [astro-ph]].

[28] J. Khoury and F. Piazza, JCAP 0907, 026 (2009) arXiv:0811.3633 [hep-th]].

[29] Y. -i. Takamizu, S. Mukohyama, M. Sasaki and Y. Tanaka, JCAP 1006, 019 (2010) arXiv:1004.1870 [astro-ph.CO]].

[30] N. Bartolo, M. Fasiello, S. Matarrese and A. Riotto, JCAP 1008, 008 (2010) arXiv:1004.0893 [astro-ph.CO]]; JCAP 1009, 035 (2010) arXiv:1006.5411 [astro-ph.CO]]; JCAP 1012, 026 (2010) arXiv:1010.3993 [astro-ph.CO]]; M. Fasiello, arXiv:1106.2189 [astro-ph.CO].

[31] C. Burrage, R. H. Ribeiro and D. Seery, JCAP 1107, 032 (2011) arXiv:1103.4126 [astro-ph.CO]]; R. H. Ribeiro and D. Seery, JCAP 1110, 027 (2011) arXiv:1108.3839 [astro-ph.CO]].

[32] M. Libanov, S. Mironov and V. Rubakov, Phys. Rev. D 84, 083502 (2011) [arXiv:1105.6230 [astro-ph.CO]].

[33] N. S. Sugiyama, E. Komatsu and T. Futamase, Phys. Rev. Lett. 106, 251301 (2011).

[34] R. H. Ribeiro, JCAP 1205, 037 (2012) arXiv:1202.4453 [astro-ph.CO]].

[35] E. Komatsu, D. N. Spergel and B. D. Wandelt, Astrophys. J. 634, 14 (2005) astro-ph/0305189.

[36] D. Babich, P. Creminelli and M. Zaldarriaga, JCAP 0408, 009 (2004) astro-ph/0405356.

[37] P. Creminelli, A. Nicolis, L. Senatore, M. Tegmark and M. Zaldarriaga, JCAP 0605, 004 (2006) astro-ph/0509029.

[38] M. Liguori, F. K. Hansen, E. Komatsu, S. Matarrese and A. Riotto, Phys. Rev. D 73, 043505 (2006) astro-ph/0509098.

[39] K. M. Smith and M. Zaldarriaga, Mon. Not. Roy. Astron. Soc. 417, 2 (2011) astro-ph/0612571.

[40] A. P. S. Yadav, E. Komatsu and B. D. Wandelt, Astrophys. J. 664, 680 (2007) astro-ph/0701921.

[41] P. Creminelli, L. Senatore and M. Zaldarriaga, JCAP 0703, 019 (2007) astro-ph/0606001.

[42] J. R. Fergusson and E. P. S. Shellard, Phys. Rev. D 80, 043510 (2009) arXiv:0812.3413 [astro-ph]].

[43] L. Senatore, K. M. Smith and M. Zaldarriaga, JCAP 1001, 028 (2010) arXiv:0905.3746 [astro-ph.CO]].

[44] P. D. Meerburg, J. P. van der Schaar and P. S. Corasaniti, JCAP 0905, 018 (2009) [arXiv:0901.4044] [hep-th]].

[45] P. Creminelli, G. D'Amico, M. Musso, J. Norena and E. Trincherini, JCAP 1102, 006 (2011) arXiv:1011.3004 [hep-th]].

[46] C. L. Bennett et al., arXiv:1212.5225 [astro-ph.CO].

[47] P. Creminelli and M. Zaldarriaga, JCAP 0410, 006 (2004) astro-ph/0407059.

[48] F. Arroja, A. E. Romano and M. Sasaki, Phys. Rev. D 84, 123503 (2011) arXiv:1106.5384 [astro-ph.CO]]; P. Adshead, C. Dvorkin, W. Hu and E. A. Lim, Phys. Rev. D 85, 023531 (2012) arXiv:1110.3050 [astro-ph.CO]].

[49] M. H. Namjoo, H. Firouzjahi and M. Sasaki, arXiv:1210.3692 [astro-ph.CO].

[50] C. Cheung, A. L. Fitzpatrick, J. Kaplan and L. Senatore, JCAP 0802, 021 (2008) arXiv:0709.0295 [hep-th]].

[51] J. Ganc and E. Komatsu, JCAP 1012, 009 (2010) arXiv:1006.5457 [astro-ph.CO]].

[52] S. Renaux-Petel, JCAP 1010, 020 (2010) arXiv:1008.0260 [astro-ph.CO]].

[53] C. Germani and Y. Watanabe, JCAP 1107, 031 (2011) [Addendum-ibid. 1107, A01 (2011)] arXiv:1106.0502 [astroph.CO]].

[54] C. Armendariz-Picon, T. Damour and V. F. Mukhanov, Phys. Lett. B 458, 209 (1999) hep-th/9904075.

[55] J. Garriga and V. F. Mukhanov, Phys. Lett. B 458, 219 (1999) hep-th/9904176.

[56] X. Chen, M. -x. Huang, S. Kachru and G. Shiu, JCAP 0701, 002 (2007) hep-th/0605045.

[57] G. W. Horndeski, Int. J. Theor. Phys. 10, 363-384 (1974).

[58] C. Deffayet, X. Gao, D. A. Steer and G. Zahariade, Phys. Rev. D 84, 064039 (2011) arXiv:1103.3260 [hep-th]].

[59] C. Charmousis, E. J. Copeland, A. Padilla and P. M. Saffin, Phys. Rev. Lett. 108, 051101 (2012) [arXiv:1106.2000 [hep-th]].

[60] T. Kobayashi, M. Yamaguchi and J. 'i. Yokoyama, Prog. Theor. Phys. 126, 511 (2011) arXiv:1105.5723 [hep-th]]. 
[61] X. Gao and D. A. Steer, JCAP 1112, 019 (2011) arXiv:1107.2642 [astro-ph.CO]].

[62] A. De Felice and S. Tsujikawa, Phys. Rev. D 84, 083504 (2011) arXiv:1107.3917 [gr-qc]].

[63] S. Mizuno and K. Koyama, Phys. Rev. D 82, 103518 (2010) arXiv:1009.0677 [hep-th]].

[64] A. De Felice and S. Tsujikawa, JCAP 1104, 029 (2011) arXiv:1103.1172 [astro-ph.CO]].

[65] T. Kobayashi, M. Yamaguchi and J. 'i. Yokoyama, Phys. Rev. D 83, 103524 (2011) arXiv:1103.1740 [hep-th]].

[66] X. Gao, T. Kobayashi, M. Yamaguchi and J. 'i. Yokoyama, Phys. Rev. Lett. 107, 211301 (2011) arXiv:1108.3513 [astroph.CO]]; X. Gao et al., arXiv:1207.0588 [astro-ph.CO].

[67] R. L. Arnowitt, S. Deser and C. W. Misner, Phys. Rev. 117, 1595 (1960).

[68] A. De Felice and S. Tsujikawa, JCAP 1202, 007 (2012) arXiv:1110.3878] [gr-qc]].

[69] S. Renaux-Petel, JCAP 1202, 020 (2012) [arXiv:1107.5020 [astro-ph.CO]].

[70] L. Senatore, K. M. Smith and M. Zaldarriaga, JCAP 1001 (2010) 028 arXiv:0905.3746 [astro-ph.CO]].

[71] T. Kobayashi, M. Yamaguchi and J. 'i. Yokoyama, Phys. Rev. Lett. 105, 231302 (2010) arXiv:1008.0603 [hep-th]].

[72] K. Kamada, T. Kobayashi, M. Yamaguchi and J. 'i. Yokoyama, Phys. Rev. D 83, 083515 (2011) arXiv:1012.4238 [astroph.CO]].

[73] A. De Felice, S. Tsujikawa, J. Elliston and R. Tavakol, JCAP 1108, 021 (2011) arXiv:1105.4685 [astro-ph.CO]].

[74] J. Ohashi and S. Tsujikawa, JCAP 1210, 035 (2012) arXiv:1207.4879 [gr-qc]].

[75] [PLANCK Collaboration], arXiv:astro-ph/0604069. 